TRANSACTIONS OF THE

AMERICAN MATHEMATICAL SOCIETY

Volume 360, Number 9, September 2008, Pages 4815-4839

S 0002-9947(08)04618-7

Article electronically published on April 11, 2008

\title{
ON CROSSED HOMOMORPHISMS ON SYMPLECTIC MAPPING CLASS GROUPS
}

\author{
RYOJI KASAGAWA
}

\begin{abstract}
For a symplectic manifold $(M, \omega)$ with a relation $Q$ between Chern classes of it and the cohomology class of the symplectic form $\omega$, we construct a crossed homomorphism $F_{Q}$ on the symplectomorphism group of $(M, \omega)$ with values in the cohomology group of $M$ and show an application to the symplectic flux group. Moreover we see that $F_{Q}$ descends to a crossed homomorphism on the symplectic mapping class group of $(M, \omega)$ and show a nontrivial example of it.
\end{abstract}

\section{INTRODUCTION}

There are a lot of works for the mapping class group $\mathcal{M}$ of an oriented surface $\Sigma$, which is the group of path components of the diffeomorphism group Dif $f_{+}(\Sigma)$ of $\Sigma$ with the $C^{\infty}$ topology. If we take an area form $\omega_{\Sigma}$ on $\Sigma$, we also obtain the group $\mathcal{S}$ of path components of the group of area preserving diffeomorphisms. By Moser [6], $\mathcal{S}$ is isomorphic to $\mathcal{M}$. Since a symplectic manifold $(M, \omega)$ is a highdimensional analogue to $\left(\Sigma, \omega_{\Sigma}\right)$, the group $S(M, \omega)$ of path components of the symplectomorphism group of $(M, \omega)$ is considered as a high-dimensional analogue to the mapping class group $\mathcal{M}$.

As works for $\mathcal{M}$, there are constructions of crossed homomorphisms from $\mathcal{M}$ to the first (co)homology group of $\Sigma$ and investigations of them by Morita 7] and by Trapp [1]. In this paper, we carry out an analogy of them in terms of differential forms. For a symplectic manifold $(M, \omega)$ with a relation $Q$ between Chern classes of $(M, \omega)$ and the cohomology class of the symplectic form $\omega$, we construct a crossed homomorphism $F_{Q}$ from the symplectomorphism group $\operatorname{Symp}(M, \omega)$ of $(M, \omega)$ to the cohomology group of $M$ using Chern-Simons forms or Bott homomorphisms. $F_{Q}$ has a connection with the flux homomorphism which is a homomorphism from the identity component of $\operatorname{Symp}(M, \omega)$ to $H^{1}(M ; \mathbb{R})$. So we can show an application of $F_{Q}$ to the flux group $\Gamma_{\omega}$ for $(M, \omega)$, which gives an extension of a result for $\Gamma_{\omega}$ by Kȩdra-Kotschick-Morita 2. Moreover we show that $F_{Q}$ descends to a crossed homomorphism $\mathcal{F}_{Q}$ from the symplectic mapping group $\mathcal{S}(M, \omega)$ to a quotient of the cohomology group of $M$ and that $\mathcal{F}_{Q}$ is generally nontrivial by giving a nontrivial example.

This paper is organized as follows. In section 2, we define our crossed homomorphisms and state the main results. In sections 3 and 4, we introduce some tools for

Received by the editors July 26, 2006.

2000 Mathematics Subject Classification. Primary 53C15, 57S05; Secondary 55R40, 57R20.

(C)2008 American Mathematical Society

Reverts to public domain 28 years from publication 4815 
the proof of Theorem A: Bott homomorphisms and a derivation formula for a curve of pushforward connections. In section 5 , we prove Theorem A. In section 6 , we give a topological definition of our crossed homomorphisms, which is needed for the computation of a nontrivial example. In section 7 , we recall Thurston's theorem for a symplectic fibration and consider diffeomorphisms on it with some properties. These are also needed for the construction of our example. In sections 8 and 9 , we show a nontrivial example of our crossed homomorphisms on the symplectic mapping class group.

\section{DeFinition of OUR CROSSED HOMOMORPHISMS AND MAIN RESUlTS}

Let $(M, \omega)$ be a closed symplectic manifold of dimension $2 n$. Let $p: \mathcal{J} \rightarrow M$ be the fiber bundle over $M$ whose fiber at each $x \in M$ is the space of positive compatible complex structures on the symplectic vector space $\left(T_{x} M, \omega_{x}\right)$. Note that $\mathcal{J}$ is a smooth manifold. A section $J: M \rightarrow \mathcal{J}$ is an $\omega$-positive compatible almost complex structure on $M$. Since the fibers of $\mathcal{J}$ are contractible, $J$ is unique up to homotopy. Hence the Chern classes of $(M, \omega)$ are defined as those of the complex vector bundle $(T M, J)$. Similarly Chern classes of a symplectic vector bundle $\left(E, \omega_{E}\right) \rightarrow N$ of $\operatorname{rank}_{\mathbb{R}}=2 n$ over a manifold $N$ are defined. For each invariant symmetric multilinear function $q_{j} \in I_{n}^{j}:=I^{j}(U(n))$ of degree $j$ on the Lie algebra $u(n)$, the associated Chern class of $\left(E, \omega_{E}\right)$ in $H^{2 j}(N ; \mathbb{R})$ is denoted by $q_{j}\left(E, \omega_{E}\right)$. For a complex vector bundle $\xi$ of $\operatorname{rank}_{\mathbb{C}}=n$, its Chern class associated with $q_{j}$ is denoted by $q_{j}(\xi)$. Let $I_{n}[w]$ be the polynomial algebra of one variable $w$ with coefficients in the invariant Weil algebra $I_{n}=\sum_{j \geqq 0} I_{n}^{j}$ of $U(n)$. Put $I_{n}[w]^{k}=$ $\sum_{j=0}^{k} I_{n}^{j} \cdot w^{k-j}$. For each $Q=\sum_{j=0}^{k} q_{j} \cdot w^{k-j} \in I_{n}[w]^{k}$ and each $a \in H^{2}(N ; \mathbb{R})$, we can consider the cohomology class $Q\left(\left(E, \omega_{E}\right), a\right)=\sum_{j=0}^{k} q_{j}\left(E, \omega_{E}\right) \cup a^{k-j} \in$ $H^{2 k}(N ; \mathbb{R})$. In particular, we put $Q(M, \omega):=Q((T M, \omega),[\omega]) \in H^{2 k}(M ; \mathbb{R})$ for the symplectic manifold $(M, \omega)$.

We assume that there exists $Q=\sum_{j=0}^{k} q_{j} \cdot w^{k-j} \in I_{n}[w]^{k}$ such that the equality

$$
Q(M, \omega)=\sum_{j=0}^{k} q_{j}(T M, \omega) \cup[\omega]^{k-j}=0
$$

holds in $H^{2 k}(M ; \mathbb{R})$. We fix such a $Q \in I_{n}[w]^{k}$.

The pullback bundle $p^{*} T M$ over $\mathcal{J}$ has a canonical hermitian structure which is given by the hermitian form $h_{J_{x}}()=,\omega_{x}\left(, J_{x}\right)-\sqrt{-1} \omega_{x}($,$) on the fiber$ $\left.p^{*} T M\right|_{J_{x}}=T_{x} M$ at each $J_{x} \in \mathcal{J}$ with $p\left(J_{x}\right)=x$. Let $\operatorname{Symp}(M, \omega)$ be the group of symplectomorphisms on $(M, \omega)$. The standard action of $\operatorname{Symp}(M, \omega)$ on $M$ lifts to ones on $\mathcal{J}$ and on $p^{*} T M$ given, respectively, by

$$
\operatorname{Symp}(M, \omega) \times \mathcal{J} \ni\left(\varphi, J_{x}\right) \mapsto \varphi\left(J_{x}\right):=d \varphi_{x} \cdot J_{x} \cdot d \varphi_{\varphi(x)}^{-1} \in \mathcal{J}_{\varphi(x)} \subset \mathcal{J}
$$

and

$$
\operatorname{Symp}(M, \omega) \times p^{*} T M \ni(\varphi, v) \mapsto d \varphi_{x}(v) \in T_{\varphi(x)} M=\left.p^{*} T M\right|_{\varphi\left(J_{x}\right)} \subset p^{*} T M,
$$

where $\left.v \in p^{*} T M\right|_{J_{x}}=T_{x} M$. The action on $p^{*} T M$ is one of $U(n)$ vector bundle isomorphisms. 
The assumption $Q(M, \omega)=0$ implies $\sum_{j=0}^{k} q_{j}\left(p^{*} T M\right) \cup\left[p^{*} \omega\right]^{k-j}=0$ in $H^{2 k}(\mathcal{J} ; \mathbb{R})$.

This means that, if we take a $U(n)$-connection $A$ on $p^{*} T M$ with curvature form $F_{A}$, there exists a $2 k-1$-form $\mu$ on $\mathcal{J}$ satisfying

$$
\sum_{j=0}^{k} q_{j}\left(F_{A}^{(j)}\right) \wedge p^{*} \omega^{k-j}+d \mu=0
$$

since $q_{j}\left(p^{*} T M\right)$ is represented by the $2 j$-form $q_{j}\left(F_{A}^{(j)}\right)=q_{j}\left(F_{A}, F_{A}, \cdots, F_{A}\right)$ by Chern-Weil theory.

We define the map

$$
\tilde{F}_{Q}: \operatorname{Symp}(M, \omega) \rightarrow \Omega^{2 k-1}(M)
$$

by

$$
\tilde{F}_{Q}(\varphi)=J^{*}\left\{\sum_{j=0}^{k} j \int_{0}^{1} q_{j}\left(\alpha, F_{A_{t}}^{(j-1)}\right) d t \wedge p^{*} \omega^{k-j}+\varphi_{*} \mu-\mu\right\},
$$

where $\alpha=\varphi_{*} A-A$ and $A_{t}=A+t \alpha$. The integrals are relative Chern-Simons forms.

Put

$$
Q^{\prime}=\sum_{j=0}^{k}(k-j) q_{j} \cdot w^{k-j-1} \in I_{n}[w]^{k-1}
$$

then we have $Q^{\prime}(M, \omega)=\sum_{j=0}^{k}(k-j) q_{j}(T M, \omega) \cup[\omega]^{k-j-1}$ in $H^{2 k-2}(M ; \mathbb{R})$.

Theorem A. For a closed symplectic 2 -manifold $(M, \omega)$ with $Q(M, \omega)=0$ for some $Q \in I_{n}[w]^{k}$, the map $F_{Q}: \operatorname{Symp}(M, \omega) \ni \varphi \mapsto\left[\tilde{F}_{Q}(\varphi)\right] \in H^{2 k-1}(M ; \mathbb{R})$ is a well-defined crossed homomorphism. Its class $\left[F_{Q}\right]$ in the group cohomology $H^{1}\left(\operatorname{Symp}(M, \omega), H^{2 k-1}(M ; \mathbb{R})\right)$ is independent of the choice of connection $A, 2 k-1$ form $\mu$ and section $J$. Moreover the equality $\frac{d}{d s} F_{Q}\left(\varphi_{s}\right)=Q^{\prime}(M, \omega) \cup\left[\iota\left(X_{s}\right) \omega\right]$ holds for any smooth path $\left\{\varphi_{s}\right\}_{s \in[0,1]}$ in $\operatorname{Symp}(M, \omega)$ with family of vector fields $X_{s}$ defined by $\frac{d}{d s} \varphi_{s}=X_{s} \circ \varphi_{s}$.

The map $F_{Q}$ is a crossed homomorphism if and only if it satisfies $F_{Q}(\varphi \psi)=$ $F_{Q}(\varphi)+\varphi_{*} F_{Q}(\psi)$ for any $\varphi, \psi \in \operatorname{Symp}(M, \omega)$, where the action of $\operatorname{Symp}(M, \omega)$ on $H^{*}(M ; \mathbb{R})$ is given by $(\varphi, a) \mapsto \varphi_{*} a:=\left(\varphi^{-1}\right)^{*} a$ for each $(\varphi, a) \in \operatorname{Symp}(M, \omega) \times$ $H^{*}(M ; \mathbb{R})$.

Let $\operatorname{Symp}_{0}(M, \omega)$ be the identity component of $\operatorname{Symp}(M, \omega)$. For any $\varphi \in$ $\operatorname{Symp}_{0}(M, \omega)$, choose a path $\varphi_{s} \in \operatorname{Symp}_{0}(M, \omega)(0 \leqq s \leqq 1)$ from $\varphi_{0}=i d$ to $\varphi_{1}=\varphi$. The flux homomorphism

$$
\text { Flux } \sim \operatorname{Symp}_{0}^{\sim}(M, \omega) \rightarrow H^{1}(M ; \mathbb{R})
$$

is defined by $F_{l u x}^{\sim}(\varphi)=\left[\int_{0}^{1} \iota\left(X_{s}\right) \omega d s\right] \in H^{1}(M ; \mathbb{R})$, where $\operatorname{Symp}_{0}^{\sim}(M, \omega)$ is the universal covering of $\operatorname{Symp}_{0}(M, \omega)$. Let $\Gamma_{\omega}$ be the subgroup of $H^{1}(M ; \mathbb{R})$ which is the image by Flux $\sim$ of the fiber of the unversal covering at the identity. $\Gamma_{\omega}$ is called the flux group. Flux $\sim$ descends to a homomorphism:

$$
\text { Flux }: \operatorname{Symp}_{0}(M, \omega) \rightarrow H^{1}(M ; \mathbb{R}) / \Gamma_{\omega},
$$

which is also called the flux homomorphism. 
If we integrate the formula for a derivative of $F_{Q}\left(\varphi_{s}\right)$ in Theorem A in the case when $\varphi_{s}$ is a path from the identity to $\varphi$, then we obtain $F_{Q}(\varphi)=Q^{\prime}(M, \omega) \cup$ $\operatorname{Flux}^{\sim}\left(\left[\varphi_{s}\right]\right)$, where $\left[\varphi_{s}\right]$ denotes the element of $\operatorname{Symp}_{0}^{\sim}(M, \omega)$ defined by the path $\varphi_{s}$. This implies $\Gamma_{\omega} \subset \operatorname{ker}\left[Q^{\prime}(M, \omega) \cup\right]$, where $Q^{\prime}(M, \omega) \cup$ is the homomorphism from $H^{1}(M ; \mathbb{R})$ to $H^{2 k-1}(M ; \mathbb{R})$ defined by the cup product with $Q^{\prime}(M, \omega)$. This result has already been given by Kędra-Kotschick-Morita [2] in the case when $[\omega]^{k}$ is proportional to a product of fundamental Chern classes of $(M, \omega)$. In the case of $k=1$, namely in the case when $[\omega]$ is proportional to the first Chern class $c_{1}(M, \omega)$, Kotschick-Morita 3 proved $\Gamma_{\omega}=\{0\}$ and that Flux extends to the whole group $\operatorname{Symp}(M, \omega)$ as a crossed homomorphism. Our crossed homomorphism $F_{Q}$ with $Q=w-\lambda c_{1}$ also gives an extension of Flux.

Corollary B. $\Gamma_{\omega} \subset \bigcap_{Q} \operatorname{ker}\left[Q^{\prime}(M, \omega) \cup\right]$, where $Q$ runs over $\bigcup_{k \geqq 0}\left\{Q \in I_{n}[w]^{k} \mid\right.$ $Q(M, \omega)=0\}$.

Since Flux is surjective and the restriction of $F_{Q}$ to $\operatorname{Symp}_{0}(M, \omega)$ factors through $H^{1}(M ; \mathbb{R}) / \Gamma_{\omega}$,

$$
\left.F_{Q}\right|_{\operatorname{Symp}_{0}(M, \omega)}: \operatorname{Symp}_{0}(M, \omega) \stackrel{F l u x}{\rightarrow} H^{1}(M ; \mathbb{R}) / \Gamma_{\omega} \stackrel{Q^{\prime}(M, \omega) \cup}{\rightarrow} H^{2 k-1}(M ; \mathbb{R}),
$$

we have the following proposition.

Proposition C. Under the assumption of Theorem $A$, if $Q^{\prime}(M, \omega) \cup: H^{1}(M ; \mathbb{R}) \rightarrow$ $H^{2 k-1}(M ; \mathbb{R})$ is a nontrivial homomorphism, then the cohomology class $\left[F_{Q}\right]$ is nonzero in $H^{1}\left(\operatorname{Symp}(M, \omega), H^{2 k-1}(M ; \mathbb{R})\right)$.

Put

$$
D_{Q}(M, \omega)=Q^{\prime}(M, \omega) \cup H^{1}(M ; \mathbb{R}) ;
$$

then it is a subgroup of $H^{2 k-1}(M ; \mathbb{R})$ invariant under the standard action by $\operatorname{Symp}(M, \omega)$. Let $\mathcal{S}(M, \omega)$ be the quotient $\operatorname{Symp}(M, \omega) / \operatorname{Symp}_{0}(M, \omega)$, which is called the symplectic mapping class group of $(M, \omega)$.

Corollary D. $F_{Q}$ descends to a crossed homomorphism

$$
\mathcal{F}_{Q}: \mathcal{S}(M, \omega) \rightarrow H^{2 k-1}(M ; \mathbb{R}) / D_{Q}(M, \omega),
$$

whose cohomology class $\left[\mathcal{F}_{Q}\right] \in H^{1}\left(\mathcal{S}(M, \omega), H^{2 k-1}(M ; \mathbb{R}) / D_{Q}(M, \omega)\right)$ is independent of the choice of connection $A, 2 k-1$ form $\mu$ and almost complex structure $J$.

Next we give a nontrivial example of the crossed homomorphism $\mathcal{F}_{Q}$. Let $\left(B=T^{2 n}=\mathbb{R}^{2 n} / \mathbb{Z}^{2 n}, \beta\right)$ be the standard symplectic torus of dimension $2 n$ with coordinates $\left(x_{i}, y_{i}\right)_{i=1}^{n}$ and symplectic form $\beta=d x_{1} \wedge d y_{1}+\cdots+d x_{n} \wedge d y_{n}$. Its cohomology ring is given by $H^{*}\left(T^{2 n} ; \mathbb{Z}\right)=\bigwedge\left(d x_{i}, d y_{i}\right)$ with abuse of notation.

Let $L_{i} \rightarrow T^{2 n}$ be a complex $U(1)$-line bundle over $T^{2 n}$ for $1 \leqq i \leqq n$ with $\gamma_{i}:=c_{1}\left(L_{i}\right)=d x_{i} \wedge d y_{i} \in H^{2}\left(T^{2 n} ; \mathbb{Z}\right)$. Put $V=L_{1} \oplus \cdots \oplus L_{n}$; then $V$ is a complex $U(n)$-vector bundle of $\operatorname{rank}_{\mathbb{C}} V=n$. Let $\pi: M=P(V) \rightarrow T^{2 n}$ be the projective bundle associated with $V$; then it is a symplectic fibration with fiber $\mathbb{C} P^{n-1}$ with the standard symplectic (Fubini-Study) form. By Thurston's theorem in [10] (see also [5] or Theorem 7.1), it is a symplectic manifold with symplectic form $\omega_{K}$ for any sufficiently large $K>0$. 
Let $\mathcal{L}$ be the Hopf line bundle associated with the projective bundle $M$. Put $\eta=-c_{1}(\mathcal{L}) ;$ then we have $H^{*}(M ; \mathbb{R})=H^{*}\left(T^{2 n} ; \mathbb{R}\right)[\eta] / \sim$ with relation $\eta^{n}+$ $c_{1}(V) \eta^{n-1}+\cdots+c_{n}(V)=0$. Put

$$
Q_{K, 2}=\bar{c}_{2}-\frac{1}{2(1-n K)^{2}}\left[n w^{2}-2 \bar{c}_{1} w+\left\{n(n-1) K^{2}-2(n-1) K+1\right\} \bar{c}_{1}^{2}\right]
$$

in $I_{2 n-1}[w]^{2}$, where $\bar{c}_{i} \in I_{2 n-1}^{i}(i=1,2)$ is the form corresponding to the $i$-th Chern class. We can show $Q_{K, 2}\left(M, \omega_{K}\right)=0, Q_{K, 2}^{\prime}=-\frac{1}{(1-n K)^{2}}\left(n w-\bar{c}_{1}\right)$ and $D_{Q_{K, 2}}\left(M, \omega_{K}\right)=\left(c_{1}-n\left[\omega_{K}\right]\right) \cup H^{1}(M ; \mathbb{R})$. By Corollary D, we have the crossed homomorphism

$$
\mathcal{F}_{Q_{2, K}}: \mathcal{S}\left(M, \omega_{K}\right) \rightarrow H^{3}(M ; \mathbb{R}) /\left(c_{1}-n\left[\omega_{K}\right]\right) \cup H^{1}(M ; \mathbb{R}),
$$

where $c_{1}=\bar{c}_{1}\left(T M, \omega_{K}\right)$.

Let $\mathcal{S}_{0}\left(M, \omega_{K}\right)$ be the subgroup of $\mathcal{S}\left(M, \omega_{K}\right)$ consisting of all elements which act trivially on $H^{*}(M ; \mathbb{R})$. The restriction of $\mathcal{F}_{Q_{K, 2}}$ to $\mathcal{S}_{0}\left(M, \omega_{K}\right)$ is a homomorphism.

Theorem E. The image of $\left[\mathcal{F}_{Q_{K, 2}}\right]$ by the induced homomorphism $H^{1}\left(\mathcal{S}\left(M, \omega_{K}\right), \mathcal{H}\right)$ $\rightarrow H^{1}\left(\mathcal{S}_{0}\left(M, \omega_{K}\right), \mathcal{H}\right)$ by the restriction, where

$$
\mathcal{H}=H^{3}(M ; \mathbb{R}) /\left(c_{1}-n\left[\omega_{K}\right]\right) \cup H^{1}(M ; \mathbb{R}),
$$

is nonzero, hence so is $\left[\mathcal{F}_{Q_{K, 2}}\right]$.

\section{BOTT HOMOMORPHISMS}

In this section we shall recall Bott homomorphisms (see [12]) which are useful for our computation.

Let $G$ be a Lie group, but we consider only $G=U(n)$ in this paper. Let $\pi: P \rightarrow M$ be a principal $G$-bundle over a manifold $M$. Let $A_{h}(h=0,1, \ldots, r)$ be $r+1$ connection forms on $P$ and $\Delta^{r}:=\left\{\left(t_{0}, t_{1}, \ldots, t_{r}\right) \in \mathbb{R}^{r+1} \mid t_{h} \geqq 0, \sum_{h=0}^{r} t_{h}=1\right\}$ the standard $r$-simplex. Then we have the average connection $\tilde{A}=\sum_{h=0}^{r} t_{h} A_{h}$ on the product principal $G$-bundle $\pi \times i d: P \times \Delta^{r} \rightarrow M \times \Delta^{r}$. Let $I^{k}(G)$ be the vector space of invariant, symmetric, multilinear functions on the $k$-th product $\mathfrak{g}^{k}$ of the Lie algebra $\mathfrak{g}$ of $G$ with values in $\mathbb{R}$. For each $c \in I^{k}(G)$, put $\Delta\left(A_{0}, \ldots, A_{r}\right) c=(-1)^{\left[\frac{r+1}{2}\right]} \int_{\Delta^{r}} c\left(F_{\tilde{A}}^{(k)}\right)$, where $F_{\tilde{A}}$ is the curvature form of $\tilde{A}$, $c\left(F_{\tilde{A}}^{(k)}\right)$ denotes $c\left(F_{\tilde{A}}, F_{\tilde{A}}, \ldots, F_{\tilde{A}}\right)$ and the orientation of $\Delta^{r}$ is given by $d t_{1} \wedge \cdots \wedge$ $d t_{r}\left(t_{0}=1-\sum_{h=1}^{r} t_{h}\right)$. Then we have the Bott homomorphism $\Delta\left(A_{0}, \ldots, A_{r}\right): I^{k}(G)$ $\rightarrow \Omega^{2 k-r}(M)$. The Bott homomorphisms have the following properties:

(1) $d \Delta\left(A_{0}, \ldots, A_{r}\right) c=\sum_{h=0}^{r}(-1)^{h} \Delta\left(A_{0}, \ldots, A_{h-1}, A_{h+1}, \ldots, A_{r}\right) c$; in particular, $d \Delta\left(A_{0}\right) c=0$ for $r=0$.

(2) $\Delta\left(A_{0}\right): I(G) \rightarrow \Omega^{*}(M)$ is the Chern-Weil homomorphism; that is, the equality $\Delta\left(A_{0}\right) c=c\left(F_{A_{0}}^{(k)}\right)$ holds.

(3) $\Delta\left(A_{0}, A_{1}\right) c=k \int_{0}^{1} c\left(\alpha, F_{A_{t}}^{(k-1)}\right) d t$, where $\alpha=A_{1}-A_{0}$ and $A_{t}=A_{0}+t \alpha$.

Let $Q$ be another principal $U(n)$-bundle over $M$ and $\tilde{\varphi}: Q \rightarrow P$ a $U(n)$-bundle isomorphism over a diffeomorphism $\varphi$ of $M$. Then the pushforward connection $\tilde{\varphi}_{*} A=\left(\tilde{\varphi}^{-1}\right)^{*} A$ can be considered.

(4) $\Delta\left(\tilde{\varphi}_{*} A_{0}, \tilde{\varphi}_{*} A_{1}, \ldots, \tilde{\varphi}_{*} A_{r}\right) c=\varphi_{*} \Delta\left(A_{0}, A_{1}, \ldots, A_{r}\right) c$, where $\varphi_{*}=\left(\varphi^{-1}\right)^{*}$. 


\section{The DERIVATIVE OF A CURVE OF PUSHFORWARD CONNECTIONS}

In this section we shall derive a derivation formula for a curve of pushforward connections.

In the setting of Theorem A, let $A$ be a $U(n)$-connection on $p^{*} T M$ and $\nabla_{A}$ : $\Omega^{0}\left(p^{*} T M\right) \rightarrow \Omega^{1}\left(p^{*} T M\right)$ the covariant derivative with respect to it. For any $\varphi \in$ $\operatorname{Symp}(M, \omega), \varphi_{*} A$ denotes the pushforward connection of $A$ by $\varphi$, which is the pullback connection $\left(\varphi^{-1}\right)^{*} A$ of $A$ by the inverse of $\varphi$.

Here and hereafter we use the same notation for the actions of $\varphi \in \operatorname{Symp}(M, \omega)$ on $M$, on $\mathcal{J}$ and on $p^{*} T M$. In terms of a covariant derivative, the pushforward connection is given by $\nabla_{\varphi_{*} A}=\varphi_{*} \nabla_{A}=\varphi_{*}\left(\nabla_{A}\left(\left(\varphi^{-1}\right)_{*}(\cdot)\right)\right)$.

Let $J_{x}$ and $K_{y}$ be two points of $\mathcal{J}$ with $p\left(J_{x}\right)=x$ and $p\left(K_{y}\right)=y$, respectively. Let $\left(U, u^{i}\right)$ and $\left(V, v^{j}\right)$ be local coordinate systems of $\mathcal{J}$ around $J_{x}$ and $K_{y}$ with orthonormal frames $e=\left(e_{1}, \ldots, e_{n}\right)$ for $\left.p^{*} T M\right|_{U}$ and $f=\left(f_{1}, \ldots, f_{n}\right)$ for $\left.p^{*} T M\right|_{V}$, respectively. We can put $\nabla_{A} e=e \otimes a$ for some $u(n)$-valued 1-form $a$ on $U$.

For $\varphi \in \operatorname{Symp}(M, \omega)$, we assume $\varphi\left(J_{x}\right)=K_{y}$ and consider it near $J_{x}$ and $K_{y}$. Put $\psi=\varphi^{-1}$. We can set $\varphi^{*} f=e \otimes \Phi^{-1}$, where $\Phi$ is a $U(n)$-valued function on $U$ near $J_{x}$. Similarly we can put $\psi^{*} e=f \otimes \Psi^{-1}$, where $\Psi$ is a $U(n)$-valued function on $V$ near $K_{y}$. Then we have $\psi^{*} \Phi=\Psi^{-1}$ and $\varphi^{*} \Psi=\Phi^{-1}$. Direct computations show the following lemma.

Lemma 4.1. In the trivialization $\left.p^{*} T M\right|_{V} \cong V \times \mathbb{C}^{n}$ near $K_{y}$ with respect to the orthonormal frame $f$, the covariant derivative with respect to $\varphi_{*} A$ is given by

$$
\nabla_{\varphi_{*} A}=d+\psi^{*}\left(-d \Phi \Phi^{-1}+\Phi a \Phi^{-1}\right)=d+\Psi^{-1} d \Psi+\Psi^{-1}\left(\psi^{*} a\right) \Psi
$$

and its curvature $F_{\varphi_{*} A}$ is given by

$$
F_{\varphi_{*} A}=\psi^{*}\left(\Phi F_{a} \Phi^{-1}\right)=\Psi^{-1} \psi^{*} F_{a} \Psi
$$

where $F_{a}=d a+a \wedge a$ is a local expression of the curvature of $A$ near $J_{x}$.

Next we compute the derivative $\frac{d}{d s}\left(\varphi_{s *} A-A\right)$ of a smooth path $\varphi_{s}$ in $\operatorname{Symp}(M, \omega)$. Let $\tilde{X}_{s}$ be the smooth family of vector fields on $\mathcal{J}$ given by $\frac{d}{d s} \varphi_{s}=$ $\tilde{X}_{s} \circ \varphi_{s}$. Hereafter we omit the parameter $s$ if it is not particularly needed. In the trivialization $\left.p^{*} T M\right|_{V} \cong V \times \mathbb{C}^{n}$ of Lemma 4.1 $\alpha=\varphi_{*} A-A$ is given by

$$
\alpha=\Psi^{-1} d \Psi+\Psi^{-1}\left(\psi^{*} a\right) \Psi-b,
$$

where $b$ is the column vector valued 1 -form defined by $\nabla_{A} f:=\left(\nabla_{A} f_{1}, \ldots, \nabla_{A} f_{n}\right)=$ $f \otimes b$. A direct computation shows that

$$
\dot{\alpha}=\frac{d}{d s} \alpha=\Psi^{-1}\left\{d\left(\dot{\Psi} \Psi^{-1}\right)+\left[\psi^{*} a, \dot{\Psi} \Psi^{-1}\right]+\frac{d}{d s}\left(\psi^{*} a\right)\right\} \Psi .
$$

To begin with, we take $\varphi_{0}=i d$ and consider the derivative at $s=0$. We can assume $e=f, b=a$ and $\Phi_{0}=\Psi_{0}=I$. Hence we have

$$
\dot{\alpha}_{0}=d \dot{\Psi}+[a, \dot{\Psi}]+\left.\frac{d}{d s}\left(\psi^{*} a\right)\right|_{s=0}=d_{a}\left\{\dot{\Psi}+\iota\left(\tilde{Y}_{0}\right) a\right\}+\iota\left(\tilde{Y}_{0}\right) F_{a},
$$

where $d_{a}=d+[a$,$] and \tilde{Y}_{s}$ is defined by $\frac{d}{d s} \psi_{s}=\tilde{Y}_{s} \circ \psi_{s}$ on $\mathcal{J}$ with $\tilde{Y}_{0}=\left.\tilde{Y}_{s}\right|_{s=0}$. We have $\tilde{Y}_{s}=-\psi_{s *} \tilde{X}_{s}$.

We note that $d_{a}$ and $F_{a}$ define the global objects $d_{A}$, which are the covariant exterior differential and $F_{A}$, respectively. It is easy to see that $\beta:=\dot{\Psi}+\iota\left(\tilde{Y}_{0}\right) a$ also 
defines a global section of the bundle $\operatorname{End}\left(p^{*} T M\right)$ over $\mathcal{J}$, which can be denoted by $\beta_{\dot{\varphi}_{0}, A}$. Therefore we get

$$
\dot{\alpha}_{0}=d_{A} \beta_{\dot{\varphi}_{0}, A}+\iota\left(\tilde{Y}_{0}\right) F_{A} .
$$

Next we consider the general case. For any smooth curve $\varphi_{s}$, put $\xi_{u}=\varphi_{u+s} \varphi_{s}^{-1}$; then we have $\xi_{0}=i d$. Since we have

$$
\frac{d}{d s}\left(\varphi_{s *} A-A\right)=\left.\frac{d}{d u}\left(\varphi_{u+s} \varphi_{s}^{-1} \varphi_{s}\right)_{*} A\right|_{u=0}=\left.\frac{d}{d u} \xi_{u *} \varphi_{s *} A\right|_{u=0},
$$

we obtain

$$
\frac{d}{d s}\left(\varphi_{s *} A-A\right)=d_{\varphi_{s *} A} \beta_{\dot{\xi}_{0}, \varphi_{s *} A}+\iota\left(\tilde{V}_{0}\right) F_{\varphi_{s *} A},
$$

where $\frac{d}{d u} \xi_{u}^{-1}=\tilde{V}_{u} \circ \xi_{u}^{-1}$. The equality $\tilde{V}_{0}=\left.\frac{d}{d u} \xi_{u}^{-1}\right|_{u=0}=-\tilde{X}_{s}$ implies the following lemma.

Lemma 4.2. Let $A$ be a $U(n)$-connection on $p^{*} T M$ and $\varphi_{s}$ a smooth curve in $\operatorname{Symp}(M, \omega)$ with a smooth family of vector fields $\tilde{X}_{s}$ on $\mathcal{J}$ defined by $\frac{d}{d s} \varphi_{s}=$ $\tilde{X}_{s} \circ \varphi_{s}$. Then the equality

$$
\frac{d}{d s}\left(\varphi_{s *} A-A\right)=d_{\varphi_{s *} A} \beta_{\dot{\xi}_{0}, \varphi_{s *} A}-\iota\left(\tilde{X}_{s}\right) F_{\varphi_{s *} A}
$$

holds for some $\beta_{\dot{\xi}_{0}, \varphi_{s *} A} \in \Gamma\left(\operatorname{End}\left(p^{*} T M\right)\right)$, where $\xi_{u}=\varphi_{u+s} \circ \varphi_{s}^{-1}$ and $\dot{\xi}_{0}=\left.\frac{d \xi_{u}}{d u}\right|_{u=0}$.

\section{Proof of Theorem A}

In this section we shall prove Theorem A.

Let $(M, \omega)$ be a closed symplectic manifold of dimension $2 n$ and fix $Q=\sum_{j} q_{j}$. $w^{k-j} \in I_{n}[w]^{k}$ satisfying $Q(M, \omega)=0$ in $H^{2 k}(M ; \mathbb{R})$. As in section 2 we have the $\operatorname{map} \tilde{F}_{Q}: \operatorname{Symp}(M, \omega) \rightarrow \Omega^{2 k-1}(M)$, which can be rewritten as

$$
\tilde{F}_{Q}(\varphi)=J^{*}\left\{\sum_{j=0}^{k} \Delta\left(A, \varphi_{*} A\right) q_{j} \wedge p^{*} \omega^{k-j}+\varphi_{*} \mu-\mu\right\}
$$

in terms of Bott homomorphisms. We note $\Delta\left(A, \varphi_{*} A\right) q_{0}=0$.

Put

$$
\tilde{f}_{Q}(\varphi)=\sum_{j=0}^{k} \Delta\left(A, \varphi_{*} A\right) q_{j} \wedge p^{*} \omega^{k-j}+\varphi_{*} \mu-\mu ;
$$

then we have $\tilde{F}_{Q}=J^{*} \circ \tilde{f}_{Q}$.

Lemma 5.1. The map $\tilde{f}_{Q}: \operatorname{Symp}(M, \omega) \rightarrow \Omega^{2 k-1}(\mathcal{J})$ induces a crossed homomorphism $f_{Q}: \operatorname{Symp}(M, \omega) \rightarrow H^{2 k-1}(\mathcal{J} ; \mathbb{R})$. Moreover the cohomology class $\left[f_{Q}\right]$ in $H^{1}\left(\operatorname{Symp}(M, \omega), H^{2 k-1}(\mathcal{J})\right)$ is independent of the choice of connection $A$ and $2 k-1$ form $\mu$.

Proof. Using the properties of Bott homomorphisms in section 3 and the definition of $\mu$, we can easily show $d \tilde{f}_{Q}(\varphi)=0$ and

$$
\tilde{f}_{Q}(\varphi \psi)-\tilde{f}_{Q}(\varphi)-\varphi_{*} \tilde{f}_{Q}(\psi)=d\left\{-\sum_{j=0}^{k} \Delta\left(A, \varphi_{*} A,(\varphi \psi)_{*} A\right) q_{j} \wedge p^{*} \omega^{k-j}\right\}
$$


for any $\varphi, \psi \in \operatorname{Symp}(M, \omega)$. The map

$$
f_{Q}: \operatorname{Symp}(M, \omega) \ni \varphi \mapsto\left[\tilde{f}_{Q}(\varphi)\right] \in H^{2 k-1}(\mathcal{J} ; \mathbb{R})
$$

turns out to be a well-defined crossed homomorphism.

Let $B$ and $\nu$ be another connection and $2 k-1$ form satisfying

$$
\sum_{j=0}^{k} \Delta(B) q_{j} \wedge p^{*} \omega^{k-j}+d \nu=0 .
$$

Put

$$
\alpha=\nu-\mu+\sum_{j=0}^{k} \Delta(A, B) q_{j} \wedge p^{*} \omega^{k-j} ;
$$

then it is easy to see that $\alpha$ is a closed $2 k-1$ form on $\mathcal{J}$.

Put

$$
\tilde{g}_{Q}(\varphi)=\sum_{j=0}^{k} \Delta\left(B, \varphi_{*} B\right) q_{j} \wedge p^{*} \omega^{k-j}+\varphi_{*} \nu-\nu
$$

for any $\varphi \in \operatorname{Symp}(M, \omega)$; then it also induces a crossed homomorphism $g_{Q}$. A computation shows

$$
\begin{aligned}
& \tilde{g}_{Q}(\varphi)-\tilde{f}_{Q}(\varphi) \\
& =\varphi_{*} \alpha-\alpha+d\left[\sum_{j=1}^{k}\left\{-\Delta\left(B, \varphi_{*} A, \varphi_{*} B\right) q_{j}+\Delta\left(A, B, \varphi_{*} A\right) q_{j}\right\} \wedge p^{*} \omega^{k-j}\right] .
\end{aligned}
$$

Therefore $\left[g_{Q}(\varphi)\right]-\left[f_{Q}(\varphi)\right]=\varphi_{*}[\alpha]-[\alpha]$ holds in $H^{2 k-1}(\mathcal{J} ; \mathbb{R})$. This shows $g_{Q}-f_{Q}=\delta \alpha$ as a 1-cochain of the group $\operatorname{Symp}(M, \omega)$ with values in $H^{2 k-1}(\mathcal{J} ; \mathbb{R})$; hence $\left[g_{Q}\right]=\left[f_{Q}\right]$ in $H^{1}\left(\operatorname{Symp}(M, \omega), H^{2 k-1}(\mathcal{J} ; \mathbb{R})\right)$.

Since any $\omega$-positive compatible almost complex structure $J: M \rightarrow \mathcal{J}$ induces the same isomorphism $H^{*}(\mathcal{J} ; \mathbb{R}) \stackrel{\cong}{\rightrightarrows} H^{*}(M ; \mathbb{R})$, we have the first part of Theorem A.

Next we prove the second part of Theorem A. We compute the derivative $\frac{d}{d s} f_{Q}\left(\varphi_{s}\right)$ of a smooth curve $\varphi_{s}$ in $\operatorname{Symp}(M, \omega)$. There is the following derivation formula for Bott homomorphisms or Chern-Simons forms in the general setting.

Let $P \rightarrow M$ be a $G$-bundle and $q \in I^{j}(G)$ an invariant polynomial of degree $j$. For a connection $\bar{\theta}$ on the product $G$-bundle $P \times I \rightarrow M \times I$ with coordinate $s \in I=[0,1]$, we consider the corresponding family $\theta_{s}=\left.\bar{\theta}\right|_{s=\text { const }}$ of connections on $P$. Let $\bar{\theta}^{\prime}$ and $\theta_{s}^{\prime}$ be another pair. Then we have

$$
\frac{\partial}{\partial s} \Delta\left(\theta_{s}, \theta_{s}^{\prime}\right) q=j\left\{q\left(\frac{\partial \theta_{s}^{\prime}}{\partial s}, F_{\theta_{s}^{\prime}}^{(j-1)}\right)-q\left(\frac{\partial \theta_{s}}{\partial s}, F_{\theta_{s}}^{(j-1)}\right)\right\}+\text { exact form }
$$

(see [12]). We apply this formula to our situation. Take $\bar{\theta}=A$ and $\bar{\theta}^{\prime}=\varphi_{s *} A$, which are connections on $p^{*} T M \times I$, where $A$ is considered as a constant along $I$. Then we have $\theta_{s}=A$, which is independent of $s$, and $\theta_{s}^{\prime}=\varphi_{s *} A$ with abuse of 
notation. Take $q=q_{j}$. In this case, the formula (5.1) and Lemma 4.2 imply

$$
\begin{aligned}
\frac{d}{d s} \Delta\left(A, \varphi_{s *} A\right) q_{j} & =j q_{j}\left(d_{\varphi_{s *} A} \beta_{\dot{\xi}_{0}, \varphi_{s *} A}-\iota\left(\tilde{X}_{s}\right) F_{\varphi_{s *} A}, F_{\varphi_{s *} A}^{(j-1)}\right)+\text { exact form } \\
& =j d q_{j}\left(\beta_{\dot{\xi}_{0}, \varphi_{s *} A}, F_{\varphi_{s *} A}^{(j-1)}\right)-\iota\left(\tilde{X}_{s}\right) q_{j}\left(F_{\varphi_{s *} A}^{(j)}\right)+\text { exact form } \\
& =-\iota\left(\tilde{X}_{s}\right) \Delta\left(\varphi_{s *} A\right) q_{j}+d R_{j},
\end{aligned}
$$

where $R_{j}$ is some $2 j-2$-form on $\mathcal{J}$ for each $j$. On the other hand, we have

$$
\frac{d}{d s} \psi_{s}^{*} \mu=\left.\frac{d}{d u}\left(\eta_{u}^{*} \psi_{s}^{*} \mu\right)\right|_{u=0}=-d\left\{\iota\left(\tilde{X}_{s}\right) \psi_{s}^{*} \mu\right\}-\iota\left(\tilde{X}_{s}\right) d\left(\psi_{s}^{*} \mu\right),
$$

where $\eta_{u}=\xi_{u}^{-1}=\varphi_{s} \varphi_{u+s}^{-1}=\psi_{s}^{-1} \psi_{s+u}$ with $\left.\frac{d}{d u} \eta_{u}\right|_{u=0}=-\tilde{X}_{s}$.

Using these formulas, properties of Bott homomorphisms and the fact that the interior product is an anti-derivative of degree -1 , we have

$$
\begin{aligned}
\frac{d}{d s} \tilde{f}_{Q}\left(\varphi_{s}\right)= & -\sum_{j=0}^{k}\left\{\iota\left(\tilde{X}_{s}\right) \Delta\left(\varphi_{s *} A\right) q_{j}-d R_{j}\right\} \wedge p^{*} \omega^{k-j}-d\left\{\iota\left(\tilde{X}_{s}\right) \psi_{s}^{*} \mu\right\}-\iota\left(\tilde{X}_{s}\right) d \psi_{s}^{*} \mu \\
=- & \sum_{j=0}^{k}\left\{\iota\left(\tilde{X}_{s}\right) \Delta\left(\varphi_{s *} A\right) q_{j}\right\} \wedge p^{*} \omega^{k-j} \\
& \quad+\iota\left(\tilde{X}_{s}\right) \psi_{s}^{*}\left\{\sum_{j=0}^{k} \Delta(A) q_{j} \wedge p^{*} \omega^{k-j}\right\}+\text { exact form } \\
= & \sum_{j=0}^{k} \Delta\left(\varphi_{s *} A\right) q_{j} \wedge \iota\left(\tilde{X}_{s}\right)\left(p^{*} \omega^{k-j}\right)+\text { exact form } \\
= & \sum_{j=0}^{k}(k-j) \Delta\left(\varphi_{s *} A\right) q_{j} \wedge p^{*} \omega^{k-j-1} \wedge \iota\left(\tilde{X}_{s}\right) p^{*} \omega+\text { exact form. }
\end{aligned}
$$

Since $\varphi_{s *} A$ is also a $U(n)$-connection on $p^{*} T M$, we obtain

$$
\frac{d}{d s} f_{Q}\left(\varphi_{s}\right)=\sum_{j=0}^{k}(k-j) q_{j}\left(p^{*} T M\right) \cup p^{*}[\omega]^{k-j-1} \cup\left[\iota\left(\tilde{X}_{s}\right) p^{*} \omega\right] .
$$

Let $X_{s}$ be the family of vector fields on $M$ defined by $\frac{d}{d s} \varphi_{s}=X_{s} \circ \varphi_{s}$; then we have $J^{*}\left(\iota\left(\tilde{X}_{s}\right) p^{*} \omega\right)=\iota\left(X_{s}\right) \omega$. Therefore $\frac{d}{d s} F_{Q}\left(\varphi_{s}\right)=J^{*} \frac{d}{d s} f_{Q}\left(\varphi_{s}\right)$ implies the following proposition.

Proposition 5.2. The equality $\frac{d}{d s} F_{Q}\left(\varphi_{s}\right)=\sum_{j=0}^{k}(k-j) q_{j}(M, \omega) \cup[\omega]^{k-j-1} \cup\left[\iota\left(X_{s}\right) \omega\right]$ holds in $H^{2 k-1}(M ; \mathbb{R})$. it.

This proposition is the second part of Theorem A, which concludes the proof of

\section{ANOTHER DEFINITION OF THE CROSSED HOMOMORPHISM}

In this section we shall define another map $h_{Q}: \operatorname{Symp}(M, \omega) \rightarrow H^{2 k-1}(M ; \mathbb{R})$ for the fixed $A$ and $\mu$ in section 2 and show $h_{Q}=-F_{Q}$.

Let $(M, \omega)$ be a closed symplectic manifold of dimension $2 n$. Fix $Q \in I_{n}[w]^{k}$ with $Q(M, \omega)=0$ in $H^{2 k}(M ; \mathbb{R})$ as before. For any $\varphi \in \operatorname{Symp}(M, \omega)$, we consider 
the mapping tori $M_{\varphi}:=[0,1] \times M /(1, x) \sim\left(0, \varphi^{-1}(x)\right)$ of $\varphi$ and $\mathcal{J}_{\varphi}$ of $\varphi: \mathcal{J} \rightarrow \mathcal{J}$. Then we have the fiber bundle $p_{\varphi}: \mathcal{J}_{\varphi} \rightarrow M_{\varphi}$. Note that $\mathcal{J}_{\varphi}$ and $M_{\varphi}$ are homotopy equivalent.

Let $p r:(-\varepsilon, 1+\varepsilon) \times M \rightarrow M_{\varphi}$ and $p r:(-\varepsilon, 1+\varepsilon) \times \mathcal{J} \rightarrow \mathcal{J}_{\varphi}$ be the obvious projections for small $\varepsilon>0$. By considering $\omega$ as a closed 2 -form on $(-\varepsilon, 1+\varepsilon) \times M$ which is constant along $(-\varepsilon, 1+\varepsilon)$, it descends to a closed 2 -form $\omega_{\varphi}$ on $M_{\varphi}$. The pullback bundle $p_{\varphi}^{*} T^{M} M_{\varphi}$ of the tangent bundle $T^{M} M_{\varphi}$ along the fibers also has the canonical hermitian structure. In particular it is a complex vector bundle.

We consider the class

$$
\bar{Q}_{\varphi}:=Q\left(\left(p_{\varphi}^{*} T^{M} M_{\varphi}, p_{\varphi}^{*} \omega_{\varphi}\right),\left[p_{\varphi}^{*} \omega_{\varphi}\right]\right)=\sum_{j=0}^{k} q_{j}\left(p_{\varphi}^{*} T^{M} M_{\varphi}, p_{\varphi}^{*} \omega_{\varphi}\right) \cup\left[p_{\varphi}^{*} \omega_{\varphi}\right]^{k-j}
$$

in $H^{2 k}\left(\mathcal{J}_{\varphi} ; \mathbb{R}\right)$. We identify $\mathcal{J}$ with the fiber at $0: \mathcal{J}=\mathcal{J} \times 0 \hookrightarrow \mathcal{J}_{\varphi}$. By the assumption $Q(M, \omega)=0$, we have $\left.\bar{Q}_{\varphi}\right|_{\mathcal{J}}=0$.

Recall the notation in section 2, $p: \mathcal{J} \rightarrow M$ is the projection and $A$ is a $U(n)$ connection on $p^{*} T M$. Put $Q\left(A, p^{*} \omega\right):=\sum_{j=0}^{k} q_{j}\left(F_{A}^{(j)}\right) \wedge p^{*} \omega^{k-j}$; then it is an exact $2 k$-form on $\mathcal{J}$. There exists $\mu \in \Omega^{2 k-1}(\mathcal{J})$ such that $d \mu+Q\left(A, p^{*} \omega\right)=0$.

Take a $U(n)$-connection $A_{\varphi}$ on $p_{\varphi}^{*} T^{M} M_{\varphi}$ and a $2 k-1$ form $\mu_{\varphi}$ on $\mathcal{J}_{\varphi}$ satisfying $\iota_{(-\varepsilon, \varepsilon)}^{*} A_{\varphi}=A$ and $\iota_{(-\varepsilon, \varepsilon)}^{*} \mu_{\varphi}=\mu$, where $\iota_{(-\varepsilon, \varepsilon)}:(-\varepsilon, \varepsilon) \times \mathcal{J} \hookrightarrow \mathcal{J}_{\varphi}$ is the inclusion and $A$ and $\mu$ are considered as a connection and as a $2 k-1$ form on $(\varepsilon, \varepsilon) \times \mathcal{J}$ which are constant along $[0,1]$. Then we have $d \mu_{\varphi}+Q\left(A_{\varphi}, p_{\varphi}^{*} \omega_{\varphi}\right)=0$ near $\mathcal{J}$. Therefore we have $\left[Q\left(A_{\varphi}, p_{\varphi}^{*} \omega_{\varphi}\right)+d \mu_{\varphi}\right] \in H^{2 k}\left(\mathcal{J}_{\varphi}, \mathcal{J} ; \mathbb{R}\right)$ whose image in $H^{2 k}\left(\mathcal{J}_{\varphi} ; \mathbb{R}\right)$ is $\bar{Q}_{\varphi}$.

We define the class $h_{Q}(\varphi)$ in $H^{2 k-1}(\mathcal{J} ; \mathbb{R})$ as the image of $\left[Q\left(A_{\varphi}, p_{\varphi}^{*} \omega_{\varphi}\right)+d \mu_{\varphi}\right]$ by the composition of isomorphisms:

$$
\begin{aligned}
& H^{2 k}\left(\mathcal{J}_{\varphi}, \mathcal{J} ; \mathbb{R}\right) \stackrel{\text { excision }}{\cong} H^{2 k}([0,1] \times \mathcal{J},\{0,1\} \times \mathcal{J} ; \mathbb{R}) \\
& \stackrel{\text { Künneth }}{\cong} H^{1}([0,1],\{0,1\} ; \mathbb{R}) \otimes H^{2 k-1}(\mathcal{J} ; \mathbb{R}) \\
& \quad \cong H^{2 k-1}(\mathcal{J} ; \mathbb{R}) .
\end{aligned}
$$

Lemma 6.1. The map

$$
h_{Q}=h_{Q, A, \mu}: \operatorname{Symp}(M, \omega) \ni \varphi \mapsto h_{Q}(\varphi) \in H^{2 k-1}(\mathcal{J} ; \mathbb{R})
$$

is well-defined, that is, it is independent of the choice of $A_{\varphi}$ and $\mu_{\varphi}$.

Proof. Let $B_{\varphi}$ and $\nu_{\varphi}$ be another connection on $p_{\varphi}^{*} T^{M} M_{\varphi}$ and the $2 k-1$ form on $\mathcal{J}_{\varphi}$ satisfying $\iota_{(-\varepsilon, \varepsilon)}^{*} B_{\varphi}=A$ and $\iota_{(-\varepsilon, \varepsilon)}^{*} \nu_{\varphi}=\mu$. Then we have $d \nu_{\varphi}+Q\left(B_{\varphi}, p_{\varphi}^{*} \omega_{\varphi}\right)=0$ near $\mathcal{J}$. We have only to show $\left[Q\left(B_{\varphi}, p_{\varphi}^{*} \omega_{\varphi}\right)+d \nu_{\varphi}\right]=\left[Q\left(A_{\varphi}, p_{\varphi}^{*} \omega_{\varphi}\right)+d \mu_{\varphi}\right]$ in $H^{2 k}\left(\mathcal{J}_{\varphi}, \mathcal{J} ; \mathbb{R}\right)$. But it follows from

$$
\begin{aligned}
Q\left(B_{\varphi}, p_{\varphi}^{*} \omega_{\varphi}\right)+d \nu_{\varphi}= & Q\left(A_{\varphi}, p_{\varphi}^{*} \omega_{\varphi}\right)+d \mu_{\varphi} \\
& +d\left\{\sum_{j=0}^{k} \Delta\left(A_{\varphi}, B_{\varphi}\right) q_{j} \wedge p_{\varphi}^{*} \omega_{\varphi}^{k-j}+\nu_{\varphi}-\mu_{\varphi}\right\}
\end{aligned}
$$

on $\mathcal{J}_{\varphi}$ and $\sum_{j=0}^{k} \Delta\left(A_{\varphi}, B_{\varphi}\right) q_{j} \wedge p_{\varphi}^{*} \omega_{\varphi}^{k-j}+\nu_{\varphi}-\mu_{\varphi} \equiv 0$ near $\mathcal{J}$.

Proposition 6.2. $f_{Q}=-h_{Q}: \operatorname{Symp}(M, \omega) \rightarrow H^{2 k-1}(\mathcal{J} ; \mathbb{R})$. 
Proof. For any sufficiently small $\varepsilon>0$, let $\lambda_{\varepsilon}:(-\varepsilon, 1+\varepsilon) \rightarrow \mathbb{R}$ be a smooth function satisfying $\left|\lambda_{\varepsilon}(t)\right|<2 \varepsilon,\left|\lambda_{\varepsilon}^{\prime}(t)\right| \leqq$ const. independent of $\varepsilon$ and

$$
\lambda_{\varepsilon}(t)= \begin{cases}-t, & t \in(-\varepsilon, \varepsilon], \\ 0, & t \in[2 \varepsilon, 1-2 \varepsilon], \\ 1-t, & t \in[1-\varepsilon, 1+\varepsilon) .\end{cases}
$$

Clearly there exists a family $\left\{\lambda_{\varepsilon}\right\}_{\varepsilon}$ of such functions.

Put $b=\varphi_{*} A-A$ on $\mathcal{J}$. The connection $\bar{A}_{\varphi}=A+\left(t+\nu_{\varepsilon}(t)\right) b$ on $(-\varepsilon, 1+\varepsilon) \times p^{*} T M$ descends to a connection $A_{\varphi}$ on $p_{\varphi}^{*} T^{M} M_{\varphi} \cong(-\varepsilon, 1+\varepsilon) \times p^{*} T M / \sim$. Similarly the 2 -form $p^{*} \omega$ on $(-\varepsilon, 1+\varepsilon) \times \mathcal{J}$ descends to the closed 2 -form $p_{\varphi}^{*} \omega_{\varphi}$. Therefore we can calculate forms on $\mathcal{J}_{\varphi}$ as those on $(-\varepsilon, 1+\varepsilon) \times \mathcal{J}$.

Let $\nabla_{A} \stackrel{\text { loc }}{=} d+a$ be a local expression of the covariant derivative associated with the connection $A$ on $\mathcal{J}$ in some local trivialization of $p^{*} T M$; then we have

$$
\nabla_{\bar{A}_{\varphi}} \stackrel{\text { loc }}{=} d^{\mathcal{J}_{\varphi}}+a+\left(t+\lambda_{\varepsilon}\right) b
$$

where $d^{\mathcal{J}_{\varphi}}$ denotes the exterior differential on $\mathcal{J}_{\varphi}$. The curvature $F_{\bar{A}_{\varphi}}$ of $\bar{A}_{\varphi}$ is given by

$$
\begin{aligned}
F_{\bar{A}_{\varphi}} & \stackrel{\text { loc }}{=} d^{\mathcal{J}_{\varphi}}\left\{a+\left(t+\lambda_{\varepsilon}\right) b\right\}+\left\{a+\left(t+\lambda_{\varepsilon}\right) b\right\} \wedge\left\{a+\left(t+\lambda_{\varepsilon}\right) b\right\} \\
& =F_{t}+d t \wedge\left(1+\lambda_{\varepsilon}^{\prime}\right) b+\lambda_{\varepsilon} B_{t}
\end{aligned}
$$

where

$$
F_{t}=F_{A+t b}^{\mathcal{J}} \stackrel{\text { loc }}{=} d^{\mathcal{J}}(a+t b)+(a+t b) \wedge(a+t b)
$$

and

$$
B_{t, \varepsilon}=d^{\mathcal{J}} b+(a+t b) \wedge b+b \wedge(a+t b)+\lambda_{\varepsilon} b \wedge b .
$$

Here $d^{\mathcal{J}}$ denotes the exterior differential on $\mathcal{J}$. For any $q_{j} \in I_{n}^{j}$, we have

$$
\begin{aligned}
q_{j}\left(F_{\bar{A}_{\varphi}}^{(j)}\right) & =q_{j}\left(\left(d t \wedge\left(1+\lambda_{\varepsilon}^{\prime}\right) b+F_{t}+\lambda_{\varepsilon} B_{t, \varepsilon}\right)^{(j)}\right) \\
& =j\left(1+\lambda_{\varepsilon}^{\prime}\right) d t \wedge q_{j}\left(b,\left(F_{t}+\lambda_{\varepsilon} B_{t, \varepsilon}\right)^{(j-1)}\right)+q\left(\left(F_{t}+\lambda_{\varepsilon} B_{t, \varepsilon}\right)^{(j)}\right) .
\end{aligned}
$$

Put $\mu_{\varphi}=\xi_{\varphi}+d t \wedge \eta$; then we have $\xi_{\varphi} \equiv \mu$ near $0 \times \mathcal{J}, \xi_{\varphi} \equiv\left(\varphi^{-1}\right)^{*} \mu$ near $1 \times \mathcal{J}, \eta \equiv 0$ near $\{0,1\} \times \mathcal{J}$ and $d^{\mathcal{J}_{\varphi}} \mu_{\varphi}=d^{\mathcal{J}} \xi_{\varphi}+d t \wedge \xi_{\varphi}^{\prime}-d t \wedge d^{\mathcal{J}} \eta$. Put $Q\left(\bar{A}_{\varphi}, p_{\varphi}^{*} \omega_{\varphi}\right)=Q^{\mathcal{J}}+d t \wedge Q^{I}$. Since the equality $Q\left(\bar{A}_{\varphi}, p_{\varphi}^{*} \omega_{\varphi}\right)+d \mu_{\varphi}=0$ holds on $(-\varepsilon, \varepsilon) \times \mathcal{J}$, we have $Q^{\mathcal{J}}+d^{\mathcal{J}} \xi_{\varphi}=0$ and $Q^{I}+\xi_{\varphi}^{\prime}-d^{\mathcal{J}} \eta=0$ on $(-\varepsilon, \varepsilon) \times \mathcal{J}$.

Note that the integration is defined by $\int_{I} \alpha=\int_{I} \beta d t$ for $I=[0,1]$ and $\alpha=\beta \wedge d t$ by our convention.

A representative of $h_{Q}(\varphi)$ is given by

$$
\begin{aligned}
& \int_{I}\left(Q\left(\bar{A}_{\varphi}, p_{\varphi}^{*} \omega_{\varphi}\right)+d \mu_{\varphi}\right)=\int_{I} d t \wedge\left(Q^{I}+\xi_{\varphi}^{\prime}-d^{\mathcal{J}} \eta\right) \\
& =\left(\int_{I} d t \wedge Q^{I}\right)-\left[\xi_{\varphi}\right]_{0}^{1}-d^{\mathcal{J}} \int_{I} d t \wedge \eta \\
& =\int_{I} d t \wedge\left\{\sum_{j=0}^{k} j\left(1+\lambda_{\varepsilon}^{\prime}\right) q_{j}\left(b,\left(F_{t}+\lambda_{\varepsilon} B_{t, \varepsilon}\right)^{(j-1)}\right) \wedge p^{*} \omega^{k-j}\right\} \\
& -\left(\varphi^{-1}\right)^{*} \mu+\mu-d^{\mathcal{J}} \int_{I} d t \wedge \eta,
\end{aligned}
$$


which implies

$h_{Q}(\varphi)=\left[\int_{I} d t \wedge\left\{\sum_{j=0}^{k} j\left(1+\lambda_{\varepsilon}^{\prime}\right) q_{j}\left(b,\left(F_{t}+\lambda_{\varepsilon} B_{t, \varepsilon}\right)^{(j-1)}\right) \wedge p^{*} \omega^{k-j}\right\}-\varphi_{*} \mu+\mu\right]$.

For any sufficiently small $\varepsilon>0$, we have $\left|\lambda_{\varepsilon}^{\prime}\right| \leqq$ const, $\left|\operatorname{supp}\left(\lambda_{\varepsilon}\right)\right| \leqq 4 \varepsilon$ and $\left|\lambda_{\varepsilon}\right| \leqq \varepsilon$, hence $\left|B_{t, \varepsilon}\right| \leqq$ const. Take the limit as $\varepsilon \rightarrow 0$; then we have

$$
\begin{aligned}
h_{Q}(\varphi) & =\left[\int_{I} d t \wedge \sum_{j=0}^{k} j q_{j}\left(b, F_{t}^{(j-1)}\right) \wedge p^{*} \omega^{k-j}-\varphi_{*} \mu+\mu\right] \\
& =-\left[\sum_{j=0}^{k} \Delta\left(A, \varphi_{*} A\right) q_{j} \wedge p^{*} \omega^{k-j}+\varphi_{*} \mu-\mu\right] \\
& =-f_{Q}(\varphi) .
\end{aligned}
$$

This finishes the proof of the proposition.

Put $Q_{\varphi}:=\sum_{j=0}^{k} q_{j}\left(T^{M} M_{\varphi}, \omega_{\varphi}\right) \cup\left[\omega_{\varphi}\right]^{k-j} \in H^{2 k}\left(M_{\varphi} ; \mathbb{R}\right)$; then the image of it by the isomorphism $p_{\varphi}^{*}: H^{*}\left(M_{\varphi} ; \mathbb{R}\right) \rightarrow H^{*}\left(\mathcal{J}_{\varphi} ; \mathbb{R}\right)$ is $\bar{Q}_{\varphi}$.

Here we recall the Leray-Hirsch theorem [9] over $\mathbb{R}$. Let $\pi: Y \rightarrow X$ be a fibration over an arcwise-connected CW-complex $X$ with fiber $F$. Assume that the cohomology group $H^{*}(F ; \mathbb{R})$ is finitely generated and that the induced homomorphism $\iota^{*}: H^{*}(Y ; \mathbb{R}) \rightarrow H^{*}(F ; \mathbb{R})$ by the inclusion $\iota: F \hookrightarrow Y$ is surjective. Then we can take a homomorphism $s: H^{*}(F ; \mathbb{R}) \rightarrow H^{*}(Y ; \mathbb{R})$ of graded modules of degree 0 such that $\iota^{*} \circ s=i d$. We can define an $\mathbb{R}$-module isomorphism $\kappa_{s}: H^{*}(X ; \mathbb{R}) \otimes H^{*}(F ; \mathbb{R}) \rightarrow H^{*}(Y ; \mathbb{R})$ by $\kappa_{s}(u \otimes v)=\pi^{*} u \cup s(v)$. We have the formula $\kappa_{s}(u \otimes v)=\pi^{*} u \cup \kappa_{s}(1 \otimes v)$.

Back to our situation, we consider the mapping torus $M_{\varphi}$ of $\varphi \in \operatorname{Symp}(M, \omega)$, which is a fiber bundle over $S^{1}$ with fiber $M$. In the case of $\varphi^{*}=i d$ on $H^{*}(M ; \mathbb{R})$, we can easily check that the induced homomorphism $\iota^{*}: H^{*}\left(M_{\varphi} ; \mathbb{R}\right) \rightarrow H^{*}(M ; \mathbb{R})$ of the inclusion $\iota: M \hookrightarrow M_{\varphi}$ is surjective. Hence we have an $\mathbb{R}$-module isomorphism

$$
\kappa_{\varphi}=\kappa_{\varphi, s}: H^{*}\left(S^{1} ; \mathbb{R}\right) \otimes H^{*}(M ; \mathbb{R}) \stackrel{\cong}{\rightrightarrows} H^{*}\left(M_{\varphi} ; \mathbb{R}\right) .
$$

The following lemma, which is a corollary of the proof of Proposition 6.2. is needed later.

Lemma 6.3. If $\varphi_{*}=i d$ on $H^{*}(M ; \mathbb{R})$, then the equalities

$$
Q_{\varphi}=\kappa_{\varphi}\left(t^{*} \otimes H_{Q}(\varphi)\right)=\kappa_{\varphi}\left(t^{*} \otimes\left(-F_{Q}(\varphi)\right)\right)
$$

hold. Here $H_{Q}:=J^{*} \circ h_{Q}: \operatorname{Symp}(M, \omega) \rightarrow H^{2 k-1}(M ; \mathbb{R})$, and $t^{*}$ is the generator of $H^{1}\left(S^{1} ; \mathbb{Z}\right) \subset H^{1}\left(S^{1}: \mathbb{R}\right)$.

Proof. By the assumption, it is easy to see that the induced homomorphism

$$
\iota^{*}: H^{*}\left(\mathcal{J}_{\varphi} ; \mathbb{R}\right) \rightarrow H^{*}(\mathcal{J} ; \mathbb{R})
$$

of the inclusion $\iota: \mathcal{J} \hookrightarrow \mathcal{J}_{\varphi}$ as a fiber is surjective. Therefore we have the short exact sequences

$$
0 \rightarrow H^{*}\left(\mathcal{J}_{\varphi}, \mathcal{J} ; \mathbb{R}\right) \rightarrow H^{*}\left(\mathcal{J}_{\varphi} ; \mathbb{R}\right) \rightarrow H^{*}(\mathcal{J} ; \mathbb{R}) \rightarrow 0
$$


On the other hand, by the Leray-Hirsch theorem, we have the $\mathbb{R}$-module isomorphism

$$
\bar{\kappa}_{\varphi}=\bar{\kappa}_{\varphi, s}: H^{*}\left(S^{1} ; \mathbb{R}\right) \otimes H^{*}(\mathcal{J} ; \mathbb{R}) \rightarrow H^{*}\left(\mathcal{J}_{\varphi} ; \mathbb{R}\right) .
$$

These imply isomorphisms

$$
H^{2 k}\left(\mathcal{J}_{\varphi}, \mathcal{J} ; \mathbb{R}\right) \cong H^{1}\left(S^{1} ; \mathbb{R}\right) \otimes H^{2 k-1}(\mathcal{J} ; \mathbb{R}) \stackrel{\bar{\kappa}_{\varphi}}{\cong} \operatorname{ker}\left[\iota^{*}: H^{2 k}\left(\mathcal{J}_{\varphi} ; \mathbb{R}\right) \rightarrow H^{2 k}(\mathcal{J} ; \mathbb{R})\right] .
$$

Under these isomorphisms, we have $\bar{Q}_{\varphi}=\bar{\kappa}_{\varphi}\left(t^{*} \otimes h_{Q}(\varphi)\right)=\bar{\kappa}_{\varphi}\left(t^{*} \otimes\left(-f_{Q}(\varphi)\right)\right)$ by the proof of Proposition 6.2. Since any section of the bundle $p_{\varphi}: \mathcal{J}_{\varphi} \rightarrow M_{\varphi}$ is homotopy equivalent, we obtain the lemma.

\section{ThuRston's THEOREM}

In this section we shall recall Thurston's theorem [10] (see also [5]) for a symplectic fibration and consider some related diffeomorphisms.

Let $\pi: M \rightarrow B$ be a symplectic fibration whose typical fiber is a symplectic manifold $(F, \sigma)$. By definition, there is an open covering $\left\{U_{\alpha}\right\}$ of $B$ with trivialization

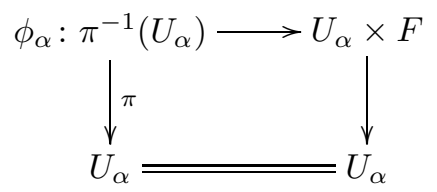

for each $U_{\alpha}$. Moreover put $\phi_{\alpha}(b)=\left.\operatorname{proj}_{2} \circ \phi_{\alpha}\right|_{F_{b}=\pi^{-1}(b)}: F_{b} \rightarrow F$ and $\phi_{\beta \alpha}(b)=$ $\phi_{\beta}(b) \circ \phi_{\alpha}(b)^{-1}$; then they define smooth maps $\phi_{\beta \alpha}: U_{\alpha} \cap U_{\beta} \rightarrow \operatorname{Symp}(F, \sigma)$. For each $b \in B$, the canonical symplectic form $\sigma_{b}$ is defined by $\sigma_{b}=\phi_{\alpha}(b)^{*} \sigma$. This form is independent of the choice of $\alpha$.

A symplectic form $\omega$ on $M$ is called compatible with the fibration $\pi$ if each fiber $\left(F_{b}, \sigma_{b}\right)$ is a symplectic submanifold of $(M, \omega)$, that is, the equality $\sigma_{b}=\iota_{b}^{*} \omega$ holds for each inclusion $\iota_{b}: F_{b} \hookrightarrow M$.

Theorem 7.1 (Thurston [10]). Let $\pi: M \rightarrow B$ be a compact symplectic fibration with symplectic fiber $(F, \sigma)$ and a connected symplectic base $(B, \beta)$, and let $\sigma_{b} \in$ $\Omega^{2}\left(F_{b}\right)$ be the canonical symplectic form on $F_{b}$. If there exists $a \in H^{2}(M)$ satisfying $\iota_{b}^{*} a=\left[\sigma_{b}\right]$ for some (and hence any) $b \in B$, then for all sufficiently large $K>0$, there exists a symplectic form $\omega_{K} \in \Omega^{2}(M)$ such that it is compatible with $\pi$ and represents the class $a+K\left[\pi^{*} \beta\right]$.

The proof of his theorem constructs a closed 2-form $\tau$ on $M$ with $[\tau]=a$ satisfying $\iota_{b}^{*} \tau=\sigma_{b}$ for all $b \in B$. The required symplectic form is given by $\omega_{K}=\tau+K \pi^{*} \beta$ for any sufficiently large $K>0$.

We fix such a $\tau$. Let $\hat{\phi}: M \rightarrow M$ be a diffeomorphism of $M$ over a symplectomorphism $\phi:(B, \beta) \rightarrow(B, \beta)$ such that its restriction $\hat{\phi}_{b}:\left(F_{b}, \sigma_{b}\right) \rightarrow\left(F_{\phi(b)}, \sigma_{\phi(b)}\right)$ to the fiber $F_{b}$ at each $b \in B$ is a symplectomorphism:

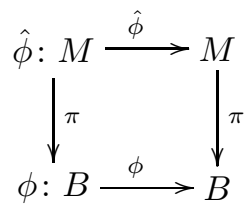

Moreover suppose that the induced homomorphism $\hat{\phi}^{*}: H^{2}(M) \rightarrow H^{2}(M)$ preserves the class $a=[\tau] \in H^{2}(M)$. 
Let Vert and Hor be the subbundles of $T M$ whose fibers at $x \in M$ are given by Vert $_{x}:=\operatorname{ker} d \pi_{x}$ and Hor $_{x}:=\left(\operatorname{ker} d \pi_{x}\right)^{\tau}:=\left\{\xi \in T_{x} M \mid \tau(\xi, \eta)=0 \forall \eta \in\right.$ Vert $\left._{x}\right\}$, respectively. Then we have the splitting $T M=V e r t \oplus H$ or and the isomorphism $d \pi_{x}: H_{\text {or }} \rightarrow T_{\pi(x)} B$ for each $x \in M$.

Put

$$
\chi_{\hat{\phi}}:=\hat{\phi}^{*} \tau-\tau
$$

then we have

$$
\hat{\phi}^{*} \omega_{K}=\omega_{K}+\chi_{\hat{\phi}}
$$

and

$$
\left.\chi_{\hat{\phi}}\right|_{V e r t} \equiv 0 .
$$

With respect to the splitting $T M=V \operatorname{Vert} \oplus \operatorname{Hor}$, the 2 -forms $\tau, \pi^{*} \beta$ and $\chi_{\hat{\phi}}$ can be represented by

$$
\tau=\left(\begin{array}{cc}
A & O \\
O & B
\end{array}\right), \quad \pi^{*} \beta=\left(\begin{array}{cc}
O & O \\
O & C
\end{array}\right) \quad \text { and } \quad \chi_{\hat{\phi}}=\left(\begin{array}{cc}
O & E \\
F & G
\end{array}\right),
$$

where $A$ and $C$ are nondegenerate. For each $t \in[0,1]$, put

$$
\Omega_{t}:=\omega_{K}+t \chi_{\hat{\phi}}=\left(\begin{array}{cc}
A & t E \\
t F & B+K C+t G
\end{array}\right) ;
$$

then we have $\Omega_{0}=\omega_{K}$ and $\Omega_{1}=\hat{\phi}^{*} \omega_{K}$. Moreover for any sufficiently large $K>$ $0, \Omega_{t}$ is nondegenerate and has the same cohomology class $\left[\Omega_{t}\right]=\left[\omega_{K}\right]$ for all $t \in[0,1]$. Therefore we can apply Moser's method to them. There is a family of diffeomorphisms $\eta_{t}$ on $M$ satisfying $\eta_{t}^{*} \Omega_{t}=\Omega_{0}=\omega_{K}$ and $\eta_{0}=i d_{M}$.

In particular we have

$$
\omega_{K}=\eta_{1}^{*} \Omega_{1}=\eta_{1}^{*} \hat{\phi}^{*} \omega_{K}=\left(\hat{\phi} \circ \eta_{1}\right)^{*} \omega_{K},
$$

which implies that the composition

$$
\Phi:=\hat{\phi} \circ \eta_{1}:\left(M, \omega_{K}\right) \rightarrow\left(M, \omega_{K}\right)
$$

is a symplectomorphism diffeotopic to $\hat{\phi}$. We have shown the following proposition.

Proposition 7.2. Under the same assumption as Thurston's theorem, let $\hat{\phi}: M \rightarrow$ $M$ be a fiberwise symplectomorphism over a symplectomorphism $\phi$ on $(B, \beta)$ with $\hat{\phi}^{*} a=a$. Then for any sufficiently large $K>0, \hat{\phi}$ is diffeotopic to a symplectomorphism $\Phi:\left(M, \omega_{K}\right) \rightarrow\left(M, \omega_{K}\right)$, where $\omega_{K}$ is the symplectic form on $M$ in Thurston's theorem.

For the symplectomorphism $\Phi$ on $\left(M, \omega_{K}\right)$ in this proposition, we consider the mapping torus $M_{\Phi}=[0,1] \times M /(0, m) \sim(1, \Phi(m))$ of $\Phi$. The closed 2-form $\omega_{K}$ defines a fiberwise symplectic form $\omega_{K, \Phi}$ on $M_{\Phi}$. Let $T^{M} M_{\Phi}$ be the tangent bundle along the fibers $M$; then the symplectic vector bundle $\left(T^{M} M_{\Phi}, \omega_{K, \Phi}\right)$ over $M_{\Phi}$ is obtained. In order to calculate the Chern classes of it, we consider a family of the mapping tori $\left\{M_{\hat{\phi} \circ \eta_{s}}\right\}_{s \in[0,1]}$ of $\left\{\hat{\phi} \circ \eta_{s}\right\}_{s \in[0,1]}$. These mapping tori are diffeomorphic to each other as fiber bundles over $S^{1}$. In particular we have the family of diffeomorphisms

induced by

$$
\xi_{s}: M_{\hat{\phi}} \rightarrow M_{\hat{\phi} \circ \eta_{s}}
$$

$$
[0,1] \times M \ni(t, m) \mapsto\left(t, \hat{\phi} \circ \eta_{s \nu(t)} \circ \hat{\phi}^{-1}(m)\right) \in[0,1] \times M,
$$


where $\nu(t)$ is a monotonically increasing function on $[0,1]$ with $\nu(t) \equiv 0$ near $t=0$ and $\nu(t) \equiv 1$ near $t=1$. Put $\xi=\xi_{1}: M_{\hat{\phi}} \rightarrow M_{\hat{\phi} \circ \eta_{1}}=M_{\Phi}$.

For each $s \in[0,1]$, the family of 2 -forms

$$
\bar{\Omega}_{s}:=\left\{\left(\hat{\phi}^{-1}\right)^{*} \Omega_{1+t(s-1)}\right\}_{t \in[0,1]}
$$

on $M$ parameterized by $t \in[0,1]$ defines a fiberwise symplectic form $\bar{\Omega}_{s}$ (we use the same symbol) on $M_{\hat{\phi} \circ \eta_{s}}$ because of

$$
\left(\hat{\phi} \circ \eta_{s}\right)^{*}\left(\hat{\phi}^{-1}\right)^{*} \Omega_{1+1(s-1)}=\eta_{s}^{*} \Omega_{s}=\omega_{K}=\left(\hat{\phi}^{-1}\right)^{*} \Omega_{1} .
$$

Let $T^{M} M_{\hat{\phi} \circ \eta_{s}}$ be the tangent bundle of $M_{\hat{\phi} \circ \eta_{s}}$ along the fibers $M$ as a fiber bundle over $S^{1}$; then the family $\left\{\left(T^{M} M_{\hat{\phi} \circ \eta_{s}}, \bar{\Omega}_{s}\right)\right\}_{s \in[0,1]}$ of symplectic vector bundles is obtained. The Chern classes of the symplectic vector bundles $\left(T^{M} M_{\hat{\phi}}, \bar{\Omega}_{0}\right)=$ $\left(T^{M} M_{\hat{\phi} \circ \eta_{0}}, \bar{\Omega}_{0}\right)$ and $\left(T^{M} M_{\Phi}, \omega_{K, \Phi}\right)=\left(T^{M} M_{\hat{\phi} \circ \eta_{1}}, \bar{\Omega}_{1}\right)$ agree under the isomorphism $\xi^{*}: H^{*}\left(M_{\Phi} ; \mathbb{R}\right) \cong H^{*}\left(M_{\hat{\phi}} ; \mathbb{R}\right)$.

Next we consider $\left(T^{M} M_{\hat{\phi}}, \bar{\Omega}_{0}\right)$. For each $t \in[0,1]$, we have

$$
\left.\bar{\Omega}_{0}\right|_{t}=\left(\hat{\phi}^{-1}\right)^{*} \Omega_{1-t}=\tau-t\left(\hat{\phi}^{-1}\right)^{*} \chi_{\hat{\phi}}+K \pi^{*} \beta .
$$

For each $\hat{m}=(t, m) \in[0,1] \times M / \sim=M_{\hat{\phi}}$, put

$$
T_{\hat{m}}^{M V} M_{\hat{\phi}}:=\text { Vert }_{m}
$$

and

$$
T_{\hat{m}}^{M H} M_{\hat{\phi}}=\left\{\xi \in T_{\hat{m}}^{M} M_{\hat{\phi}} \mid\left(\tau-t\left(\hat{\phi}^{-1}\right)^{*} \chi_{\hat{\phi}}\right)(\xi, \eta)=0 \text { for } \forall \eta \in T_{\hat{m}}^{M V} M_{\hat{\phi}}\right\} .
$$

Since $\hat{\phi}$ is a fiber mapping, we have $\left(\hat{\phi}^{-1}\right)^{*} V$ ert $\subset$ Vert. The 2 -form $\tau-t\left(\hat{\phi}^{-1}\right)^{*} \chi_{\hat{\phi}}$ is nondegenerate on Vert for each $t$ because of $\chi_{\hat{\phi}} \mid$ Vert $\equiv 0$. Therefore we have the splitting

$$
T^{M} M_{\hat{\phi}}=T^{M V} M_{\hat{\phi}} \oplus T^{M H} M_{\hat{\phi}},
$$

where $T^{M V} M_{\hat{\phi}}=\coprod_{\hat{m} \in M_{\hat{\phi}}} T_{\hat{m}}^{M V} M_{\hat{\phi}}$ and $T^{M H} M_{\hat{\phi}}=\coprod_{\hat{m} \in M_{\hat{\phi}}} T_{\hat{m}}^{M H} M_{\hat{\phi}}$ are subbundles of $T^{M} M_{\hat{\phi}}$.

With respect to the above splitting, we can take matrix expressions

$$
\tau-t\left(\hat{\phi}^{-1}\right)^{*} \chi_{\hat{\phi}}=\left(\begin{array}{cc}
A^{\prime} & O \\
O & B^{\prime}
\end{array}\right) \text { and } \pi^{*} \beta=\left(\begin{array}{cc}
O & O \\
O & C^{\prime}
\end{array}\right),
$$

where $A^{\prime}$ and $C^{\prime}$ are nondegenerate; we then have

$$
\bar{\Omega}_{0}=\tau-t\left(\hat{\phi}^{-1}\right)^{*} \chi_{\hat{\phi}}+K \pi^{*} \beta=\left(\begin{array}{cc}
A^{\prime} & O \\
O & B^{\prime}+K C^{\prime}
\end{array}\right) .
$$

Note that $T^{M V} M_{\hat{\phi}}$ is a symplectic vector bundle over $M_{\hat{\phi}}$ with symplectic structure $\left.\left(\tau-t\left(\hat{\phi}^{-1}\right)^{*} \chi_{\hat{\phi}}\right)\right|_{T^{M V} M_{\hat{\phi}}}=\left.\tau\right|_{T^{M V} M_{\hat{\phi}}}$, which is nothing but the canonical symplectic structures $\left\{\sigma_{b}\right\}_{b \in B}$ and is denoted by $\sigma_{\hat{\phi}}$. This symplectic vector bundle is denoted by $\left(T^{M V} M_{\hat{\phi}}, \sigma_{\hat{\phi}}\right)$. The mapping torus $B_{\phi}$ of $\phi$ is also a symplectic fiber bundle over $S^{1}$ with fiber $(B, \beta)$. Its tangent bundle along the fibers is also a symplectic vector bundle $\left(T^{B} B_{\phi}, \beta_{\phi}\right)$. Let $\sigma_{\hat{\phi}} \oplus \pi^{*} \beta_{\phi}$ be the symplectic structure on the vector bundle $T^{M} M_{\hat{\phi}}$ induced from $\sigma_{\hat{\phi}}$ and $\beta_{\phi}$ by the isomorphism $T^{M} M_{\hat{\phi}} \cong T^{M V} M_{\hat{\phi}} \oplus \pi^{*} T^{B} B_{\phi}$; then we have a symplectic vector bundle $\left(T^{M} M_{\hat{\phi}}, \sigma_{\hat{\phi}} \oplus \pi^{*} \beta_{\phi}\right)$. Let $J^{M V}$ be a positive compatible complex structure of $\left(T^{M V} M_{\hat{\phi}}, \sigma_{\hat{\phi}}\right)$ and let $J_{B_{\phi}}^{B}$ be a fiberwise positive 
compatible complex structure of $\left(B_{\phi}, \beta_{\phi}\right)$; then a complex structure $J$ of $T^{M} M_{\hat{\phi}}$ is induced from $J^{M V} \oplus \pi^{*} J_{B_{\phi}}^{B}$ by $T^{M} M_{\hat{\phi}} \cong T^{M V} M_{\hat{\phi}} \oplus \pi^{*} T^{B} B_{\phi} . J$ is compatible to the symplectic form $\sigma_{\hat{\phi}} \oplus \pi^{*} \beta_{\phi}$.

Lemma 7.3. For any sufficiently large $K>0$, the complex structure $J$ on $T^{M} M_{\hat{\phi}}$ is $\bar{\Omega}_{0}$-tame, i.e. $\bar{\Omega}_{0}(, J)>0$.

Proof. From the matrix representation of $\bar{\Omega}_{0}$ above, we have

$$
K>>0 \Rightarrow\left(B^{\prime}+K C^{\prime}\right)\left(\cdot, \pi^{*} J_{B_{\phi}}^{B}(\cdot)\right)>0 \Leftrightarrow \bar{\Omega}_{0}(\cdot, J(\cdot))>0 .
$$

This implies the lemma.

By this lemma, the Chern classes of $\left(T^{M} M_{\hat{\phi}}, \bar{\Omega}_{0}\right)$ agree with those of $\left(T^{M} M_{\hat{\phi}}, \sigma_{\hat{\phi}}\right.$ $\left.\oplus \pi^{*} \beta_{\phi}\right)$. Since the Chern classes of $\left(T^{M} M_{\Phi}, \omega_{K, \Phi}\right)$ and $\left(T^{M} M_{\hat{\phi}}, \bar{\Omega}_{0}\right)$ also agree under the isomorphism $\xi^{*}$, we have the following corollary.

Corollary 7.4. The Chern classes of $\left(T^{M} M_{\Phi}, \omega_{K, \Phi}\right)$ agree with those of $\left(T^{M} M_{\hat{\phi}}\right.$, $\left.\sigma_{\hat{\phi}} \oplus \pi^{*} \beta_{\phi}\right)$ under the isomorphism $\xi^{*}: H^{*}\left(M_{\Phi} ; \mathbb{R}\right) \cong H^{*}\left(M_{\hat{\phi}} ; \mathbb{R}\right)$ for any sufficiently large $K>0$.

Next we assume $\hat{\phi}=i d$ on $H^{*}(M ; \mathbb{R})$. In this case, we have isomorphisms (6.1) $\kappa_{\varphi}: H^{*}\left(S^{1} ; \mathbb{R}\right) \otimes H^{*}(M ; \mathbb{R}) \stackrel{\cong}{\rightrightarrows} H^{*}\left(M_{\varphi} ; \mathbb{R}\right)(\varphi=\hat{\phi}, \Phi)$. Put $\Xi=\kappa_{\hat{\phi}}^{-1} \circ \xi^{*} \circ \kappa_{\Phi} ;$ then we have the following commutative diagram:

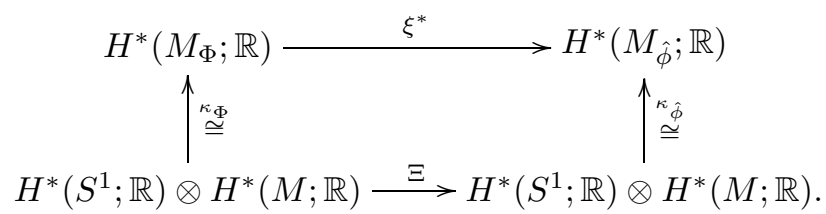

Since the restriction of $\xi$ to the fiber $M=0 \times M$ at $0 \in S^{1}$ is the identity, the matrix representation of $\Xi$ of $j$-dimension with respect to the splitting $H^{j}(M ; \mathbb{R}) \oplus$ $H^{1}\left(S^{1} ; \mathbb{R}\right) \otimes H^{j-1}(M ; \mathbb{R})$ (whose elements are considered as column vectors) is given by

$$
\Xi_{j}=\left(\begin{array}{cc}
i d & 0 \\
* & i d
\end{array}\right)
$$

Therefore, by Lemma 6.3, we have

$$
\xi^{*} Q_{\Phi}=\xi^{*} \kappa_{\Phi}\left(t^{*} \otimes\left(-F_{Q}(\Phi)\right)\right)=\kappa_{\hat{\phi}} \Xi\left(t^{*} \otimes\left(-F_{Q}(\Phi)\right)\right)=\kappa_{\hat{\phi}}\left(t^{*} \otimes\left(-F_{Q}(\Phi)\right)\right) .
$$

On the other hand, it is easy to see that we can put $\kappa_{\hat{\phi}}^{-1} \xi^{*}\left[\omega_{K, \Phi}\right]=\left[\omega_{K}\right]+t^{*} \otimes a$ for some $a \in H^{1}(M ; \mathbb{R})$, where $t^{*} \in H^{1}\left(S^{1} ; \mathbb{Z}\right) \subset H^{1}\left(S^{1} ; \mathbb{R}\right)$ is the generator. We have

$$
\xi^{*}\left[\omega_{K, \Phi}\right]=\kappa_{\hat{\phi}}\left(\left[\omega_{K}\right]+t^{*} \otimes a\right)=\kappa_{\hat{\phi}}\left[\omega_{K}\right]+\pi^{*} t^{*} \cup \kappa_{\hat{\phi}} a,
$$

hence

$$
\left(\xi^{*}\left[\omega_{K, \Phi}\right]\right)^{k-j}=\left(\kappa_{\hat{\phi}}\left[\omega_{K}\right]\right)^{k-j}+(k-j)\left(\kappa_{\hat{\phi}}\left[\omega_{K}\right]\right)^{k-j-1} \cup \pi^{*} t^{*} \cup \kappa_{\hat{\phi}} a .
$$


By Corollary 7.4, we have

$$
\begin{aligned}
\xi^{*} Q_{\Phi}= & \xi^{*}\left(\sum_{j=0}^{k} q_{j}\left(T^{M} M_{\Phi}, \omega_{K, \Phi}\right) \cup\left[\omega_{K, \Phi}\right]^{k-j}\right) \\
= & \sum_{j=0}^{k} q_{j}\left(T^{M} M_{\hat{\phi}}, \sigma_{\hat{\phi}} \oplus \pi^{*} \beta_{\phi}\right) \cup\left(\xi^{*}\left[\omega_{K, \Phi}\right]\right)^{k-j} \\
= & \sum_{j=0}^{k} q_{j}\left(T^{M} M_{\hat{\phi}}, \sigma_{\hat{\phi}} \oplus \pi^{*} \beta_{\phi}\right) \cup\left(\kappa_{\hat{\phi}}\left[\omega_{K}\right]\right)^{k-j} \\
& \quad+\pi^{*} t^{*} \cup \sum_{j=0}^{k}(k-j) q_{j}\left(T^{M} M_{\hat{\phi}}, \sigma_{\hat{\phi}} \oplus \pi^{*} \beta_{\phi}\right) \cup\left(\kappa_{\hat{\phi}}\left[\omega_{K}\right]\right)^{k-j-1} \cup \kappa_{\hat{\phi}} a .
\end{aligned}
$$

In general, the equality $\pi^{*} t^{*} \cup x=\kappa_{\hat{\phi}}\left(t^{*} \otimes \iota^{*} x\right)$ holds for any $x \in H^{j}\left(M_{\hat{\phi}} ; \mathbb{R}\right)$, where $\iota: M=M \times 0 \hookrightarrow M_{\hat{\phi}}$ is the inclusion.

Using this fact, we have

$$
\begin{aligned}
& \pi^{*} t^{*} \cup \sum_{j=0}^{k}(k-j) q_{j}\left(T^{M} M_{\hat{\phi}}, \sigma_{\hat{\phi}} \oplus \pi^{*} \beta_{\phi}\right) \cup\left(\kappa_{\hat{\phi}}\left[\omega_{K}\right]\right)^{k-j-1} \cup \kappa_{\hat{\phi}} a \\
= & \left.\kappa_{\hat{\phi}}\left(t^{*} \otimes \iota^{*}\left(\sum_{j=0}^{k}(k-j) q_{j}\left(T^{M} M_{\hat{\phi}}, \sigma_{\hat{\phi}} \oplus \pi^{*} \beta_{\phi}\right) \cup\left(\kappa_{\hat{\phi}}\left[\omega_{K}\right]\right)^{k-j-1} \cup \kappa_{\hat{\phi}} a\right)\right)\right) \\
= & \kappa_{\hat{\phi}}\left(t^{*} \otimes\left(\sum_{j=0}^{k}(k-j) q_{j}\left(T M, \omega_{K}\right) \cup\left[\omega_{K}\right]^{k-j-1} \cup a\right)\right) .
\end{aligned}
$$

Here we note $\iota^{*} q_{j}\left(T^{M} M_{\hat{\phi}}, \sigma_{\hat{\phi}} \oplus \pi^{*} \beta_{\phi}\right)=q_{j}\left(T M, \omega_{1}\right)=q_{j}\left(T M, \omega_{K}\right)$ for all $K>0$ since we can take the same almost complex structure compatible to $\omega_{K}$ for all $K>0$. Recall our notation:

$$
Q\left(\left(T^{M} M_{\hat{\phi}}, \sigma_{\hat{\phi}} \oplus \pi^{*} \beta_{\phi}\right), \kappa_{\hat{\phi}}\left[\omega_{K}\right]\right)=\sum_{j=0}^{k} q_{j}\left(T^{M} M_{\hat{\phi}}, \sigma_{\hat{\phi}} \oplus \pi^{*} \beta_{\phi}\right) \cup\left(\kappa_{\hat{\phi}}\left[\omega_{K}\right]\right)^{k-j} .
$$

Putting these equalities together, we have

$$
\begin{aligned}
& \kappa_{\hat{\phi}}\left(t^{*} \otimes\left(-F_{Q}(\Phi)\right)\right)=\xi^{*} Q_{\Phi} \\
& =Q\left(\left(T^{M} M_{\hat{\phi}}, \sigma_{\hat{\phi}} \oplus \pi^{*} \beta_{\phi}\right), \kappa_{\hat{\phi}}\left[\omega_{K}\right]\right) \\
& \quad+\kappa_{\hat{\phi}}\left(t^{*} \otimes\left(\sum_{j=0}^{k}(k-j) q_{j}\left(T M, \omega_{K}\right) \cup\left[\omega_{K}\right]^{k-j-1} \cup a\right)\right),
\end{aligned}
$$

which implies the following proposition.

Proposition 7.5. $\kappa_{\hat{\phi}}\left(t^{*} \otimes\left(-F_{Q}(\Phi)\right)\right) \equiv Q\left(\left(T^{M} M_{\hat{\phi}}, \sigma_{\hat{\phi}} \oplus \pi^{*} \beta_{\phi}\right), \kappa_{\hat{\phi}}\left[\omega_{K}\right]\right)$ in $H^{1}\left(S^{1} ; \mathbb{R}\right) \otimes H^{2 k-1}(M ; \mathbb{R})$ modulo $\kappa_{\hat{\phi}}\left(t^{*} \otimes D_{Q}\left(M, \omega_{K}\right)\right)$. 


\section{The FiRst Chern Class of A LINE BUNDle OVER A TORUS}

In this section we shall consider the first Chern class of a complex $U(1)$-line bundle over a torus in preparation for our example.

Let $L$ be a complex $U(1)$-line bundle over a torus $T^{b}$ of dimension $b$. Let $h^{U}: T^{b} \rightarrow U(1)$ be a smooth map; then it defines the $U(1)$-automorphism

$$
h: L \supset L_{x} \ni u \mapsto h^{U}(x) u \in L_{x} \subset L
$$

of $L$ over the identity. Let $L_{h}$ be the mapping torus of $h$; then it is a complex $U(1)$-bundle over $S^{1} \times T^{b}=T^{b+1}$. Let $t^{*} \in H^{1}\left(S^{1} ; \mathbb{Z}\right)$ be the generator. Let

$$
\kappa: H^{*}\left(S^{1} ; \mathbb{R}\right) \otimes H^{*}\left(T^{b} ; \mathbb{R}\right) \rightarrow H^{*}\left(S^{1} \times T^{b} ; \mathbb{R}\right)
$$

be the isomorphism in Künneth's theorem, which can also be considered as the isomorphism (6.1) with $\varphi=i d$ and $s=p^{*}: H^{*}\left(T^{b} ; \mathbb{R}\right) \rightarrow H^{*}\left(S^{1} \times T^{b} ; \mathbb{R}\right)$, where $p: S^{1} \times T^{b} \rightarrow T^{b}$ is the projection.

For simplicity, we consider elements of $H^{*}\left(S^{1} ; \mathbb{R}\right)$ and $H^{*}\left(T^{b} ; \mathbb{R}\right)$ as sitting in $H^{*}\left(S^{1} \times T^{b} ; \mathbb{R}\right)$ in the obvious way. In the case of $b=1$, we have

$$
c_{1}\left(L_{h}\right)=\kappa\left(t^{*} \otimes\left(h^{U}\right)^{*} u\right)=t^{*} \cup\left(h^{U}\right)^{*} u,
$$

where $u \in H^{1}(U(1) ; \mathbb{Z})$ is the generator. Using this fact, it is easy to see the following lemma for any $b \geqq 1$.

\section{Lemma 8.1.}

$$
c_{1}\left(L_{h}\right)=\kappa\left(t^{*} \otimes\left(h^{U}\right)^{*} u+1 \otimes c_{1}(L)\right)=t^{*} \cup\left(h^{U}\right)^{*} u+c_{1}(L) \in H^{2}\left(S^{1} \times T^{b} ; \mathbb{R}\right) .
$$

Next we consider $U(1)$-line bundles $L_{i} \rightarrow T^{b}(1 \leqq i \leqq n)$ and their direct sum $V=L_{1} \oplus \cdots \oplus L_{n}$ over $T^{b}$. Let $h_{i}: L_{i} \rightarrow L_{i}$ be the $U(1)$-automorphism defined as above by a smooth map $h_{i}^{U}: T^{b} \rightarrow U(1)$ for $1 \leqq i \leqq n$. Then we have the $U(n)$-bundle automorphism $h=h_{1} \oplus \cdots \oplus h_{n}$ of $V=L_{1} \oplus \cdots \oplus L_{n}$. The mapping torus $V_{h}$ of $h$ is identified with the direct sum $V_{h}=\left(L_{1}\right)_{h_{1}} \oplus \cdots \oplus\left(L_{n}\right)_{h_{n}}$ of the mapping tori $\left(L_{i}\right)_{h_{i}}$.

Let $\pi: P(V) \rightarrow T^{b}$ and $\pi_{h}: P\left(V_{h}\right) \rightarrow S^{1} \times T^{b}$ be the projective bundles associated with $V$ and $V_{h}$, respectively. Let $\mathcal{L} \rightarrow P(V)$ and $\tilde{\mathcal{L}}_{h} \rightarrow P\left(V_{h}\right)$ be the Hopf line bundles. The automorphism $h$ on $V$ induces a fiber map $\hat{h}$ on $P(V)$ and a $U(1)$ automorphism $\tilde{h}$ on $\mathcal{L}$ over $\hat{h}$. The mapping torus $\mathcal{L}_{\tilde{h}}$ of $\tilde{h}$ is a line bundle over the mapping torus $P(V)_{\hat{h}}$ of $\hat{h}$.
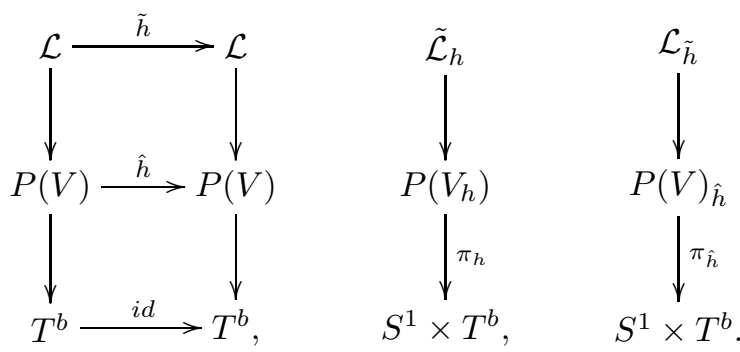

Clearly the following lemma is true. 
Lemma 8.2. $P(V)_{\hat{h}} \cong P\left(V_{h}\right)$ as $\mathbb{C} P^{n-1}$-bundles and $\mathcal{L}_{\tilde{h}} \cong \tilde{\mathcal{L}}_{h}$ as line bundles.

\section{An eXAmple}

In this section we shall give the nontriviality of our crossed homomorphisms on the symplectic mapping class group, which is the proof of Theorem E.

Let $B=T^{2 n}=\mathbb{R}^{2 n} / \mathbb{Z}^{2 n}$ be the standard $2 n$-dimensional torus with the standard coordinates $\left(x_{i}, y_{i}\right)_{i=1}^{n}$ and the standard symplectic structure $\beta=d x_{1} \wedge d y_{1}+\cdots+$ $d x_{n} \wedge d y_{n}$ for $n \geqq 3$. Its cohomology ring is given by $H^{*}\left(T^{2 n} ; \mathbb{Z}\right)=\bigwedge\left(d x_{i}, d y_{i}\right)$ with abuse of notation.

Let $L_{i} \rightarrow T^{2 n}$ be a complex $U(1)$-line bundle over $T^{2 n}$ with first Chern class $\gamma_{i}:=c_{1}\left(L_{i}\right)=d x_{i} \wedge d y_{i} \in H^{2}\left(T^{2 n} ; \mathbb{Z}\right)$ for $1 \leqq i \leqq n$. Then the direct sum $V=L_{1} \oplus \cdots \oplus L_{n} \rightarrow T^{2 n}$ is a complex vector bundle with total Chern class

$$
c(V)=\prod_{i=1}^{n} c\left(L_{i}\right)=\prod_{i=1}^{n}\left(1+\gamma_{i}\right)=e^{\beta},
$$

where $\beta$ also denotes the cohomology class of $\beta$. Hereafter we use the same symbol for a cohomology class and its representative differential form and omit the symbol $\cup$ of the cup product in cohomological computation. Let $\pi: M=P(V) \rightarrow T^{2 n}$ be the projective bundle associated with $V$; then it is a symplectic fibration with fiber $\mathbb{C} P^{n-1}$ with the Fubini-Study form. Let $\mathcal{L} \rightarrow M$ be the Hopf line bundle associated with the projective bundle $M$. Put

$$
\eta=c_{1}\left(\mathcal{L}^{*}\right)=-c_{1}(\mathcal{L}) \in H^{2}(M ; \mathbb{Z}) ;
$$

then the cohomology group of $M$ is the $H^{*}\left(T^{2 n} ; \mathbb{R}\right)$-module

$$
H^{*}(M ; \mathbb{R})=H^{*}\left(T^{2 n} ; \mathbb{R}\right)[\eta] / \sim
$$

with relation

$$
\eta^{n}+c_{1}(V) \eta^{n-1}+\cdots+c_{n}(V)=0 .
$$

The tangent bundle $T^{\mathbb{C} P} M$ along the fibers is obtained by the exact sequence

$$
0 \rightarrow \underline{\mathbb{C}} \rightarrow \mathcal{L}^{*} \otimes \pi^{*} V \rightarrow T^{\mathbb{C} P} M \rightarrow 0
$$

of vector bundles, which implies

$$
c_{1}\left(T^{\mathbb{C} P} M\right)=n \eta+\pi^{*} \beta
$$

hence

$$
\iota_{b}^{*} \eta=\frac{1}{n} \iota_{b}^{*} c_{1}\left(T^{\mathbb{C} P} M\right)
$$

for any $b \in B$. Therefore $\eta \in H^{2}(M ; \mathbb{R})$ can be taken as the class $a$ in Thurston's theorem (Theorem 7.1) for the symplectic fibration $M$ (if necessary the symplectic forms on fibers are multiplied by a common constant). By the proof of Thurston's theorem, there exists a closed 2-form $\tau$ on $M$ satisfying $[\tau]=a=\eta$ and $\iota_{b}^{*} \tau=\sigma_{b}$ for all $b \in B$. Moreover for any sufficiently large $K>0, \omega_{K}=\tau+K \pi^{*} \beta$ is a symplectic form on $M$. Because of $c\left(T^{2 n}, \beta\right)=1$, the total Chern class of $\left(M, \omega_{K}\right)$ 
is computed as

$$
\begin{aligned}
c\left(M, \omega_{K}\right) & =c\left(T^{\mathbb{C} P} M\right)=c\left(\bigoplus_{i=1}^{n} \mathcal{L} \otimes \pi^{*} L_{i}\right) \\
& =\prod_{i=1}^{n}\left(1+\eta+\gamma_{i}\right) \\
& =(1+\eta)^{n}+(1+\eta)^{n-1} \sigma_{1}+(1+\eta)^{n-2} \sigma_{2}+\cdots+\sigma_{n},
\end{aligned}
$$

where $\sigma_{i}$ is the $i$-th fundamental symmetric polynomial of $\gamma_{1}, \ldots, \gamma_{n}$ for $1 \leqq i \leqq n$.

Put $s_{j}=\sum_{i=1}^{n} \gamma_{i}^{j}$; then we have

$$
s_{1}=\sigma_{1}=\beta, \quad s_{2}=s_{3}=\cdots=0
$$

because of $\gamma_{i}^{2}=0$ for all $i$. By using Newton's formulas which are given by

$$
\left\{\begin{array}{lr}
s_{j}-\sigma_{1} s_{j-1}+\sigma_{2} s_{j-2}-\cdots+(-1)^{j-1} \sigma_{j-1} s_{1}+(-1)^{j} j \sigma_{j}=0 & (j=1, \ldots, n) \\
s_{j}-\sigma_{1} s_{j-1}+\cdots+(-1)^{j} \sigma_{n} s_{j-n}=0 & (j=n+1, n+2, \ldots),
\end{array}\right.
$$

we obtain

$$
\sigma_{j-1} s_{1}-j \sigma_{j}=0 \quad(j=1, \ldots, n),
$$

hence

$$
\sigma_{j}=\frac{1}{j !} \sigma_{1}^{j}=\frac{1}{j !} \beta^{j} \quad(j=1, \ldots, n) .
$$

Since we have $\beta^{j}=0$ for all $j \geqq n+1$, we obtain

$$
c\left(M, \omega_{K}\right)=\sum_{j=0}^{n}(1+\eta)^{n-j} \frac{1}{j !} \beta^{j}=(1+\eta)^{n} \exp \frac{\beta}{1+\eta} .
$$

Since $\pi^{*}: H^{*}\left(T^{2 n} ; \mathbb{R}\right) \rightarrow H^{*}(M ; \mathbb{R})$ is injective, hereafter we shall omit $\pi^{*}$ and write $\beta$ instead of $\pi^{*} \beta$, etc., so we have

$$
\omega_{K}=\eta+K \beta
$$

and

$$
c_{1}:=c_{1}\left(M, \omega_{K}\right)=n \eta+\sum_{i=1}^{n} \gamma_{i}=n \eta+\beta,
$$

hence

$$
\eta=\frac{\omega_{K}-K c_{1}}{1-n K} \quad \text { and } \quad \beta=\frac{-n \omega_{K}+c_{1}}{1-n K}
$$

in $H^{*}(M ; \mathbb{R})$. We can eliminate $\eta$ and $\beta$ from the formula (9.1):

$$
c\left(M, \omega_{K}\right)=\left(1+\frac{\omega_{K}-K c_{1}}{1-n K}\right)^{n} \exp \frac{c_{1}-n \omega_{K}}{1-n K+\omega_{K}-K c_{1}} .
$$

Put

$$
\begin{aligned}
Q_{K} & :=\sum_{k \geqq 0} Q_{K, k}:=\sum_{k \geqq 0} \bar{c}_{k}-(1+\bar{\eta})^{n} \exp \frac{\bar{\beta}}{1+\bar{\eta}} \\
& =\sum_{k \geqq 0} \bar{c}_{k}-\left(1+\frac{w-K \bar{c}_{1}}{1-n K}\right)^{n} \exp \frac{\bar{c}_{1}-n w}{1-n K+w-K \bar{c}_{1}}
\end{aligned}
$$


in $\sum_{k \geqq 0} I_{2 n-1}[w]^{k}$, where $\bar{c}_{i}(i \geqq 0)$ is the corresponding multilinear form to the $i$-th Chern class, $\bar{\eta}=\frac{w-K \bar{c}_{1}}{1-n K}, \bar{\beta}=\frac{-n w+\bar{c}_{1}}{1-n K}$ and $Q_{K, k} \in I_{2 n-1}[w]^{k}$ for all $k \geqq 0$. Then we have $Q_{K, 0}=Q_{K, 1}=0$ and

$$
\begin{aligned}
& Q_{K}^{\prime}:=\sum_{k \geqq 0} Q_{K, k}^{\prime}:=\frac{\bar{\beta}}{1-n K}(1+\bar{\eta})^{n-2} \exp \frac{\bar{\beta}}{1+\bar{\eta}} \\
& =\frac{\bar{\beta}}{1-n K}\left[1+\{(n-2) \bar{\eta}+\bar{\beta}\}+\left\{\frac{(n-2)(n-3)}{2 !} \bar{\eta}^{2}+(n-3) \bar{\eta} \bar{\beta}+\frac{1}{2 !} \bar{\beta}^{2}\right\}+\cdots\right] .
\end{aligned}
$$

Consequently, for any sufficiently large $K>0$, we have the symplectic manifold $\left(M, \omega_{K}\right)$ with the relations $Q_{K, k}\left(M, \omega_{K}\right)=0(k=2, \ldots, 2 n-1)$ and the crossed homomorphisms

$$
\mathcal{F}_{Q_{K, k}}: \mathcal{S}\left(M, \omega_{K}\right) \rightarrow H^{2 k-1}(M ; \mathbb{R}) / D_{Q_{K, k}}\left(M, \omega_{K}\right)
$$

by Theorem A.

Next we show that these crossed homomorphisms are nontrivial for large $K>0$. As in section 8, let $h: V=L_{1} \oplus \cdots \oplus L_{n} \rightarrow V$ be the $U(n)$-bundle isomorphism over the identify of $T^{2 n}$ corresponding to a smooth map

$$
h^{U}=\left(\begin{array}{ccc}
h_{1}^{U} & & O \\
& \ddots & \\
O & & h_{n}^{U}
\end{array}\right): T^{2 n} \rightarrow U(n)
$$

with $h_{i}^{U}: T^{2 n} \rightarrow U(1)(1 \leqq i \leqq n)$. The induced fiber map on $M=P(V)$ is denoted by

$$
\hat{h}: M \rightarrow M,
$$

whose restriction to each fiber is a symplectomorphism.

Lemma 9.1. $\hat{h}^{*}=i d$ on $H^{*}(M ; \mathbb{R})$.

Proof. The cohomology group of $M$ is given by $H^{*}(M ; \mathbb{R})=H^{*}\left(T^{2 n} ; \mathbb{R}\right)[\eta] / \sim$. Since the bundle isomorphism $h$ on $V$ naturally induces a $U(1)$-bundle isomorphism $\tilde{h}: \mathcal{L} \rightarrow \mathcal{L}$ over $\hat{h}$, we have an isomorphism $\tilde{h}^{*} \mathcal{L} \cong \mathcal{L}$ over the identity on $M$, which implies $\hat{h}^{*} \eta=\eta$. Since $\hat{h}$ is a fiber preserving map over the identity of $T^{2 n}$, we have $\hat{h}^{*}=i d$.

By Proposition 17.2, the map $\hat{h}$ is diffeotopic to a symplectomorphism $\Phi$ on $\left(M, \omega_{K}\right)$ :

$$
\hat{h} \simeq \Phi:\left(M, \omega_{K}\right) \rightarrow\left(M, \omega_{K}\right)
$$

for any sufficiently large $K>0$.

Next we compute $\mathcal{F}_{Q_{K, k}}(\Phi)$ using Proposition 7.5. Our setting is $\hat{\phi}=\hat{h}, \phi=$ $i d_{T^{2 n}}$ in Proposition 7.5 , Put $y_{i}=h_{i}^{U *} u$ and $Y=\sum_{i=1}^{n} y_{i}$ in $H^{1}\left(T^{2 n} ; \mathbb{R}\right)$. Let $\tilde{\eta}=c_{1}\left(\mathcal{L}_{\tilde{h}}^{*}\right) \in H^{2}\left(M_{\hat{h}} ; \mathbb{R}\right)$ be the first Chern class of the dual line bundle to $\mathcal{L}_{\tilde{h}}$. We consider the $\mathbb{R}$-isomorphism (6.1) $\kappa_{\hat{h}}: H^{*}\left(S^{1} ; \mathbb{R}\right) \otimes H^{*}(M ; \mathbb{R}) \stackrel{\cong}{\rightrightarrows} H^{*}\left(M_{\hat{h}} ; \mathbb{R}\right)$ with the homomorphism

$$
\begin{aligned}
s: H^{*}(M ; \mathbb{R})=H^{*}\left(T^{2 n} ; \mathbb{R}\right)[\eta] / \sim \ni a \cdot \eta^{n} & \\
& \mapsto\left(p^{*} a\right) \cdot \tilde{\eta}^{n} \in H^{*}\left(M_{\hat{h}} ; \mathbb{R}\right)=H^{*}\left(S^{1} \times T^{2 n} ; \mathbb{R}\right)[\tilde{\eta}] / \sim,
\end{aligned}
$$


where $p: S^{1} \times T^{2 n} \rightarrow T^{2 n}$ is the projection. We have the following commutative diagram:

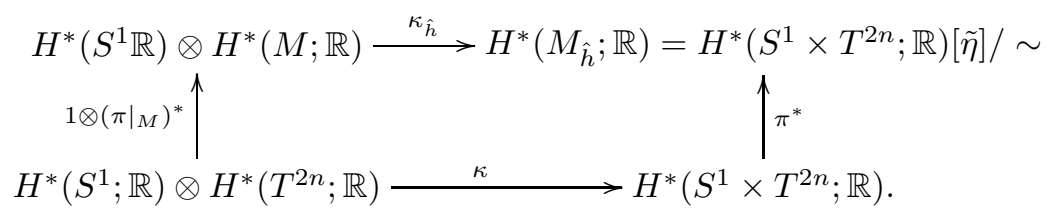

Similarly as before, elements of $H^{*}\left(S^{1} ; \mathbb{R}\right)$ and $H^{*}\left(T^{2 n} ; \mathbb{R}\right)$ are considered as sitting in $H^{*}\left(M_{\hat{h}} ; \mathbb{R}\right)$ in the obvious way, so we have

$$
H^{*}\left(S^{1} ; \mathbb{R}\right) \otimes H^{*}\left(T^{2 n} ; \mathbb{R}\right) \ni t^{*} \otimes a \mapsto t^{*} a\left(=t^{*} \cup a\right) \in H^{*}\left(M_{\hat{h}} ; \mathbb{R}\right)
$$

in the diagram above.

Put $\hat{\omega}_{K}:=\kappa_{\hat{h}}\left(\omega_{K}\right) \in H^{2}\left(M_{\hat{h}} ; \mathbb{R}\right)$; then we have

$$
\hat{\omega}_{K}=\tilde{\eta}+K \beta
$$

because of $\omega_{K}=\eta+K \beta$ in $H^{2}(M ; \mathbb{R})$.

Next we compute

$$
\begin{aligned}
& Q_{K}\left(\left(T^{M} M_{\hat{h}}, \sigma_{\hat{h}} \oplus \pi^{*} \beta_{i d}\right), \hat{\omega}_{K}\right) \\
= & c\left(T^{M} M_{\hat{h}}, \sigma_{\hat{h}} \oplus \pi^{*} \beta_{i d}\right)-\left(1+\frac{\hat{\omega}_{K}-K \hat{c}_{1}}{1-n K}\right)^{n} \exp \frac{\hat{c}_{1}-n \hat{\omega}_{K}}{1-n K+\hat{\omega}_{K}-K \hat{c}_{1}},
\end{aligned}
$$

where $\hat{c}_{1}=c_{1}\left(T^{M} M_{\hat{h}}, \sigma_{\hat{h}} \oplus \pi^{*} \beta_{i d}\right)$. Using Lemma8.1 and an isomorphism $T^{M} M_{\hat{h}} \cong$ $T^{\mathbb{C} P} M_{\hat{h}} \oplus \pi^{*} T^{B} B_{i d}$, where $T^{B} B_{i d}=\left(T T^{2 n}\right) \times S^{1}$ is considered as a trivial $U(n)$ bundle, we have

$$
\begin{aligned}
c\left(T^{M} M_{\hat{h}}, \sigma_{\hat{h}} \oplus \pi^{*} \beta_{i d}\right) & =c\left(T^{\mathbb{C} P} M_{\hat{h}} \oplus \pi^{*} T^{B} B_{i d}\right)=c\left(T^{\mathbb{C} P} M_{\hat{h}}\right) \\
& =c\left(\mathcal{L}_{\tilde{h}} \otimes \pi_{h}^{*} V_{h}\right)=c\left(\bigoplus_{i=1}^{n} \mathcal{L}_{\tilde{h}} \otimes \pi_{h}^{*}\left(L_{i}\right)_{h_{i}}\right) \\
& =\prod_{i=1}^{n}\left\{1+\tilde{\eta}+t^{*} \cup h_{i}^{U *} u+c_{1}\left(L_{i}\right)\right\} \\
& =\prod_{i=1}^{n}\left(1+\tilde{\eta}+t^{*} y_{i}+\gamma_{i}\right)
\end{aligned}
$$

in $H^{*}\left(M_{\hat{h}} ; \mathbb{R}\right)=H^{*}\left(S^{1} \times T^{2 n} ; \mathbb{R}\right)[\tilde{\eta}] / \sim$. In particular, we have

$$
\hat{c}_{1}=n \tilde{\eta}+t^{*} \cup Y+\beta \text {. }
$$

Put

$$
\zeta_{i}=t^{*} y_{i}+\gamma_{i}
$$

then we have

$$
\zeta_{i}^{2}=\left(t^{*} y_{i}+\gamma_{i}\right)^{2}=2 t^{*} y_{i} \gamma_{i}
$$

and

$$
\zeta_{i}^{3}=\cdots=\zeta_{i}^{j}=\cdots=0(j \geqq 3) .
$$

Let $\bar{\sigma}_{i}=\bar{\sigma}_{i}\left(\zeta_{1}, \ldots, \zeta_{n}\right)$ be the $i$-th fundamental symmetric polynomial of $\zeta_{j}$ 's for $1 \leqq i \leqq n$. Put $\bar{s}_{\nu}=\sum_{j=1}^{n} \zeta_{j}^{\nu}$; then Newton's formulas imply

$$
\bar{\sigma}_{\nu-2} \bar{s}_{2}-\bar{\sigma}_{\nu-1} \bar{s}_{1}+\nu \bar{\sigma}_{\nu}=0
$$


for $\nu=1, \ldots, n$, where $\bar{\sigma}_{-1}=0$ and $\bar{\sigma}_{0}=1$. This equality also holds for $\nu=$ $n+1, n+2, \ldots$ in $H^{*}\left(T^{2 n} ; \mathbb{R}\right)$.

Put

$$
f(t)=\sum_{\nu=0}^{\infty} \bar{\sigma}_{\nu} t^{\nu}=\bar{\sigma}_{0}+\bar{\sigma}_{1} t+\cdots+\bar{\sigma}_{\nu} t^{\nu}+\cdots, f(0)=\bar{\sigma}_{0}=1
$$

then we have

$$
f^{\prime}(t)-\bar{s}_{1} f(t)+\bar{s}_{2} t f(t)=\sum_{\nu=1}^{\infty}\left(\bar{\sigma}_{\nu} \nu-\bar{s}_{1} \bar{\sigma}_{\nu-1}+\bar{s}_{2} \bar{\sigma}_{\nu-2}\right) t^{\nu-1}=0 .
$$

This differential equation with $f(0)=1$ is solved as

$$
f(t)=\exp \left(\bar{s}_{1} t-\bar{s}_{2} \frac{t^{2}}{2}\right) \text {. }
$$

Since we have

$$
\begin{aligned}
& \bar{s}_{1}=\sum_{i=1}^{n} \zeta_{i}=\sum_{i=1}^{n}\left(t^{*} y_{i}+\gamma_{i}\right)=t^{*} Y+\beta, \\
& \bar{s}_{2}=\sum_{i=1}^{n} \zeta_{i}^{2}=\sum_{i=1}^{n} 2 t^{*} y_{i} \gamma_{i}=2 t^{*} Z,
\end{aligned}
$$

where

$$
Z=\sum_{i=1}^{n} y_{i} \gamma_{i}
$$

we obtain

$$
\begin{aligned}
& \prod_{i=1}^{n}\left(1+\tilde{\eta}+t^{*} y_{i}+\gamma_{i}\right)=\prod_{i=1}^{n}\left(1+\tilde{\eta}+\zeta_{i}\right) \\
& =(1+\tilde{\eta})^{n}+(1+\tilde{\eta})^{n-1} \bar{\sigma}_{1}+\cdots+(1+\tilde{\eta}) \bar{\sigma}_{n-1}+\bar{\sigma}_{n} \\
& =(1+\tilde{\eta})^{n} f\left(\frac{1}{1+\tilde{\eta}}\right) \\
& =(1+\tilde{\eta})^{n} \exp \left(\frac{t^{*} Y+\beta}{1+\tilde{\eta}}-\frac{t^{*} Z}{(1+\tilde{\eta})^{2}}\right) \\
& =(1+\tilde{\eta})^{n-2} \exp \frac{\beta}{1+\tilde{\eta}}\left[(1+\tilde{\eta})^{2}+t^{*}\{(1+\tilde{\eta}) Y-Z\}\right] .
\end{aligned}
$$

On the other hand, we have

$$
\begin{aligned}
& \left(1+\frac{\hat{\omega}_{K}-K \hat{c}_{1}}{1-n K}\right)^{n} \exp \frac{\hat{c}_{1}-n \hat{\omega}_{K}}{1-n K+\hat{\omega}_{K}-K \hat{c}_{1}} \\
= & \left(1+\tilde{\eta}-\frac{K}{1-n K} t^{*} Y\right)^{n} \exp \frac{t^{*} Y+(1-n K) \beta}{1-n K+(1-n K) \tilde{\eta}-K t^{*} Y} \\
= & (1+\tilde{\eta})^{n-2} \exp \frac{\beta}{1+\tilde{\eta}} \cdot\left((1+\tilde{\eta})^{2}+\frac{K \beta t^{*} Y}{1-n K}+(1+\tilde{\eta}) t^{*} Y\right),
\end{aligned}
$$


where we particularly used $\left(t^{*}\right)^{2}=0$. Summing up the above computation, we have

$$
\begin{aligned}
& Q_{K}\left(\left(T^{M} M_{\hat{h}}, \sigma_{\hat{h}} \oplus \pi^{*} \beta_{i d}\right), \hat{\omega}_{K}\right) \\
= & (1+\tilde{\eta})^{n-2} \exp \frac{\beta}{1+\tilde{\eta}}\left[(1+\tilde{\eta})^{2}+t^{*}\{(1+\tilde{\eta}) Y-Z\}\right] \\
& \quad-(1+\tilde{\eta})^{n-2} \exp \frac{\beta}{1+\tilde{\eta}} \cdot\left((1+\tilde{\eta})^{2}+\frac{K \beta t^{*} Y}{1-n K}+(1+\tilde{\eta}) t^{*} Y\right) \\
= & -t^{*}(1+\tilde{\eta})^{n-2} \exp \frac{\beta}{1+\tilde{\eta}} \cdot\left(Z+\frac{K}{1-n K} \beta Y\right)
\end{aligned}
$$

in $H^{*}\left(M_{\hat{h}} ; \mathbb{R}\right)$.

By Proposition 7.5 we obtain

$$
\begin{aligned}
&-\sum_{k \geqq 0} F_{Q_{K, k}}(\Phi) \equiv \iota^{*}\left\{-(1+\tilde{\eta})^{n-2} \exp \frac{\beta}{1+\tilde{\eta}} \cdot\left(Z+\frac{K}{1-n K} \beta Y\right)\right\} \quad \bmod \bigoplus_{k \geqq 0} D_{Q_{K, k}}\left(M, \omega_{K}\right) \\
&=-(1+\eta)^{n-2} \exp \frac{\beta}{1+\eta} \cdot\left(Z+\frac{K}{1-n K} \beta Y\right) .
\end{aligned}
$$

Put

$$
(1+\eta)^{n-2} \exp \frac{\beta}{1+\eta}=a_{0}+a_{2}+a_{4}+\cdots,
$$

where $a_{2 k} \in H^{2 k}(M ; \mathbb{R})$; then we have

$$
\mathcal{F}_{Q_{K, k}}([\Phi])=\left[a_{2 k-4}\left(Z+\frac{K}{1-n K} \beta Y\right)\right]=\left[a_{2 k-4} Z\right]
$$

in $H^{2 k-1}(M ; \mathbb{R}) / D_{Q_{K, k}}\left(M, \omega_{K}\right)=H^{2 k-1}(M ; \mathbb{R}) / a_{2 k-4} \beta \cdot H^{1}(M ; \mathbb{R})$. In the case of $k=2$, it is given by

$$
\begin{aligned}
\mathcal{F}_{Q_{K, 2}}: \mathcal{S}(M, \omega) \rightarrow & H^{3}(M ; \mathbb{R}) / \beta \cdot H^{1}(M ; \mathbb{R}) \\
& \cong\left\{H^{3}\left(T^{2 n} ; \mathbb{R}\right) / \beta \cdot H^{1}\left(T^{2 n} ; \mathbb{R}\right)\right\} \oplus H^{1}\left(T^{2 n} ; \mathbb{R}\right) \cdot \eta
\end{aligned}
$$

with

$$
\mathcal{F}_{Q_{K, 2}}([\Phi])=[Z]=\left[\sum_{i=1}^{n} y_{i} \gamma_{i}\right]=\left[\sum_{i=1}^{n}\left(h_{i}^{U}\right)^{*} u \gamma_{i}\right] .
$$

Moreover we have $\mathcal{F}_{Q_{K, k}}=a_{2 k-4} \mathcal{F}_{Q_{K, 2}}$ for $k \geqq 2$, where $a_{2 k-4}$ is considered as a well-defined homomorphism from $H^{3}(M ; \mathbb{R}) / D_{Q_{K, 2}}\left(M, \omega_{K}\right)$ to $H^{2 k-1}(M ; \mathbb{R}) /$ $D_{Q_{K, k}}\left(M, \omega_{K}\right)$. Since there is no condition for the maps $h_{i}^{U}(1 \leqq i \leqq n)$ and the corresponding class $[\Phi]$ belongs to $\mathcal{S}_{0}(M, \omega)$ by Lemma 9.1, it is easy to see that the restriction of $\mathcal{F}_{Q_{K, 2}}$ to $\mathcal{S}_{0}(M, \omega)$ is a nontrivial homomorphism by (9.2). Therefore we have $\iota^{*}\left[\mathcal{F}_{Q_{K, 2}}\right] \neq 0$ in $H^{1}\left(\mathcal{S}_{0}\left(M, \omega_{K}\right), H^{3}(M ; \mathbb{R}) / D_{Q_{K, 2}}\left(M, \omega_{K}\right)\right)$, hence $\left[\mathcal{F}_{Q_{K, 2}}\right] \neq 0$ in $H^{1}\left(\mathcal{S}\left(M, \omega_{K}\right), H^{3}(M ; \mathbb{R}) / D_{Q_{K, 2}}\left(M, \omega_{K}\right)\right)$. Consequently we have Theorem E. 


\section{REFERENCES}

1. K. S. Brown, Cohomology of groups, Springer, Berlin, Heidelberg, New York, Springer 1982. MR672956 (83k:20002)

2. J. Kędra, D. Kotschick and S. Morita, Crossed flux homomorphisms and vanishing theorems for flux groups, Geom. Funct. Anal. 16(2006), no. 6 1246-1273. MR2276539

3. D. Kotschick and S. Morita, Signatures of foliated surface bundles and the symplectomorphism groups of surfaces, Topology 44(2005), no. 1, 131-149. MR2104005 (2005k:57050)

4. D. Kotschick and S. Morita, Characteristic classes of foliated surface bundles with areapreserving holonomy, J. Differential Geom. 75(2007), no. 2, 273-302. MR.2286823

5. D. McDuff and D. Salamon, Introduction to Symplectic Topology, Oxford Mathematical Monographs, Oxford Univ. Press, 1995. MR1373431 (97b:58062)

6. J.K. Moser, On the volume elements on a manifold, Trans. Amer. Math. Soc., 120(1965), 286-294. MR0182927 (32:409)

7. S. Morita, Casson invariant, signature defect of framed manifolds and the secondary characteristic classes of surface bundles, J. Differential Geometry 47(1997), 560-599. MR1617632 (99h:57029)

8. A. Reznikov, Characteristic classes in symplectic topology, Selecta Math. 3(1997), 601-642. MR.1613528 (2000f:53116)

9. E. H. Spanier, Algebraic Topology, McGraw-Hill, 1966. MR.0210112 (35:1007)

10. W. Thurston, Some simple examples of symplectic manifolds, Proceedings of the American Mathematical Society, 55(1976), 467-468. MR0402764 (53:6578)

11. R. Trapp, A linear representation of the mapping class group $\mathcal{M}$ and the theory of winding numbers, Topology and its Appl. 43(1992), 47-64. MR1141372 (92k:57027)

12. I. Vaisman, Symplectic Geometry and Secondary Characteristic Classes, Progress in Math. 72, Birkhäuser, Boston, Basel, 1987. MR932470 (89f:58062)

Department of Mathematics, College of Science and Technology, Nihon University, 1-8 Kanda, Surugadai, Chiyoda-ku, Tokyo 101-8308, Japan

E-mail address: kasagawa@math.cst.nihon-u.ac.jp 